\title{
Optimal Shapes and Masses, and Optimal Transportation Problems
}

\author{
Giuseppe Buttazzo ${ }^{1}$ and Luigi De Pascale ${ }^{2}$ \\ 1 Dipartimento di Matematica, Università di Pisa \\ Via Buonarroti 2, 56127 Pisa, ITALY \\ buttazzo@dm.unipi.it \\ 2 Dipartimento di Matematica Applicata, Università di Pisa \\ Via Bonanno Pisano 25/b, 56126 Pisa, ITALY \\ depascal@dm.unipi.it
}

\begin{abstract}
Summary. We present here a survey on some shape optimization problems that received particular attention in the last years. In particular, we discuss a class of problems, that we call mass optimization problems, where one wants to find the distribution of a given amount of mass which optimizes a given cost functional. The relation with mass transportation problems will be discussed, and several open problems will be presented.
\end{abstract}

Keywords. Shape optimization, mass transportation, Monge-Kantorovich equation.

MSC 2000. 49Q10, 49J45, 74P05.

\section{Introduction}

The purpose of these notes is to give a survey on some problems in shape and mass optimization that received a lot of attention in the mathematical literature in the recent years. After a presentation of shape optimization problems in a quite general framework we give some examples that nowadays can be considered classic.

A shape optimization problem is a minimization problem where the unknown variable runs over a class of domains; then every shape optimization problem can be written in the form

$$
\min \{F(A): A \in \mathcal{A}\}
$$

where $\mathcal{A}$ is the class of admissible domains and $F$ is the cost function that one has to minimize over $\mathcal{A}$. It has to be noticed that the class $\mathcal{A}$ of admissible 
domains does not have any linear or convex structure, so in shape optimization problems it is meaningless to speak of convex functionals and similar notions. Moreover, even if several topologies on families of domains are available, in general there is not an a priori choice of a topology in order to apply the direct methods of the calculus of variations for obtaining the existence of at least an optimal domain.

We shall not give here a detailed presentation of the many problems and results in this very wide field, but we limit ourselves to discuss some model problems. We refer the reader interested in a deeper knowledge and analysis of this fascinating field to one of the several books on the subject ([3], [114], [142], [146]), to the notes by L. Tartar [149], or to the recent collection of lecture notes by D. Bucur and G. Buttazzo [37].

In many situations, a shape (or also a mass) optimization problem can be seen as an optimal control problem, where the state variable solves a PDE of elliptic type, and the control variable is given by the unknown domain (or mass distribution). We want to stress that, as it also happens in other kinds of optimal control problems, in several situations an optimal domain does not exist; this is mainly due to the fact that in these cases the minimizing sequences are highly oscillating and converge to a limit object only in a "relaxed" sense. Then we may have, in these cases, only the existence of a "relaxed solution", suitably defined, that in general is not a domain, and whose characterization may change from problem to problem.

A general procedure to relax optimal control problems can be successfully developed (see for instance [18], [60]) by using the $\Gamma$-convergence scheme which provides the right topology to use for sequences of admissible controls. In particular, for shape optimization problems, this provides the right notion of convergence for sequences of domains. However, if in the minimization problem (1), either the class $\mathcal{A}$ of admissible domains or the cost functional $F$ constrain the admissible domains to fulfill some sufficiently strong geometrical conditions, then the existence of an optimal domain may be obtained. We shall see some examples where these situations occur.

As it happens in all optimization problems, the qualitative description of the optimal solutions of a shape optimization problems, is usually obtained by means of the so called necessary conditions of optimality. These conditions have to be derived from the comparison of the cost of an optimal solution $A_{\text {opt }}$ with the cost of other suitable admissible choices, close enough to $A_{\text {opt }}$. This procedure is what is usually called a variation near the solution. We want to stress that in shape and mass optimization problems, the notion of neighbourhood is not always a priori clear; the possibility of choosing a domain variation could then be rather wide, and this often provides several necessary conditions of optimality.

In general, explicit computations of optimal shapes and masses are difficult to obtain, and one should develop efficient numerical schemes to produce approximated solutions; we will not enter this important field and we refer the interested reader to some recent books and papers (see references). 
The strict relation between some mass optimization problems and optimal transportation results has been recently shown by Bouchitté and Buttazzo in [23]; we shall shortly summarize the results obtained and we shall present some new challenging problems.

\section{Some classical problems}

In this section we present some classical examples of shape optimization problems that can be written in the form (1).

\subsection{The isoperimetric problem}

The isoperimetric problem is certainly one of the oldest shape optimization problems; given a closed set $Q \subset \mathbb{R}^{N}$, the constraint set, it consists in finding, among all Borel subsets $A \subset Q$, the one which minimizes the perimeter, once its Lebesgue measure, or more generally the quantity $\int_{A} f(x) d x$ for a given function $f \in L_{\text {loc }}^{1}\left(\mathbb{R}^{N}\right)$, is prescribed. With this notation the isoperimetric problem can be then formulated in the form (1) if we take

$$
\begin{aligned}
& F(A)=\operatorname{Per}(A), \\
& \mathcal{A}=\left\{A \subset Q: \int_{A} f(x) d x=c\right\} .
\end{aligned}
$$

Here the perimeter of a Borel set $A$ is defined as usual by

$$
\operatorname{Per}(A)=\int\left|D 1_{A}\right|=\mathcal{H}^{N-1}\left(\partial^{*} A\right)
$$

where $D 1_{A}$ is the distributional derivative of the characteristic function of $A, \mathcal{H}^{N-1}$ is the $N-1$ dimensional Hausdorff measure, and $\partial^{*} A$ is the reduced boundary of $A$ in the sense of geometric measure theory. By using the properties of the $B V$ spaces, when $Q$ is bounded we obtain the lower semicontinuity and the coercivity of the perimeter for the $L^{1}$ convergence; this enables us to apply the direct methods of the calculus of variations and to obtain straightforward the existence of an optimal solution for the problem

$$
\min \left\{\operatorname{Per}(A): A \subset Q, \int_{A} f d x=c\right\} .
$$

It is also very simple to show that in general the problem above may have no solution if we drop the assumption that $Q$ is bounded (see for instance $[16],[37])$. On the other hand, it is very well known that the classical isoperimetric problem, with $Q=\mathbb{R}^{N}$ and $f \equiv 1$, admits a solution which is any ball of measure $c$, even if the complete proof of this fact requires very delicate tools, especially when the dimension $N$ is larger than 2. A complete characterization of pairs $(Q, f)$ which provide the existence of a solution for the problem (2) seems to be difficult. 
Assume now that a set $A$ be a solution of problem (2) and that $A$ is regular enough to perform all the operations we need. Therefore we have that, at least locally, the boundary $\partial A$ can be represented by the graph of a function $u(x)$, where $x$ varies in a small neighbourhood $\omega$. By performing now the usual first variation argument with the functional

$$
\int_{\omega} \sqrt{1+|\nabla u|^{2}} d x
$$

we obtain that the function $u$ must satisfy the partial differential equation

$$
-\operatorname{div}\left(\frac{\nabla u}{\sqrt{1+|\nabla u|^{2}}}\right)=\text { constant } \quad \text { in } \omega .
$$

The term $-\operatorname{div}\left(\nabla u / \sqrt{1+|\nabla u|^{2}}\right)$ represents the mean curvature of $\partial A$ written in Cartesian coordinates; therefore we have found for a regular solution $A$ of the isoperimetric problem (2) the following necessary condition of optimality:

the mean curvature of $\partial A$ is constant in the interior points of $Q$.

Actually, the regularity of $\partial A$ does not need to be assumed as a hypothesis but is a consequence of some suitable conditions on the datum $f$ (regularity theory).

A variant of the isoperimetric problem consists in not counting some parts of the boundary $\partial A$ in the cost functional. More precisely, if $Q$ is the closure of an open set $\Omega$ with a Lipschitz boundary, we may consider problem (2) with $\operatorname{Per}(A)$ replaced by the cost functional

$$
\operatorname{Per}_{\Omega}(A)=\int_{\Omega}\left|D 1_{A}\right|=\mathcal{H}^{N-1}\left(\Omega \cap \partial^{*} A\right) .
$$

The existence of a solution when $\Omega$ is bounded still holds, as above, together with nonexistence examples when this boundedness condition is dropped.

\subsection{The Newton's problem of optimal aerodynamical profiles}

The problem of finding the best aerodynamical profile for a body in a fluid stream under some constraints on its size is another classical question which can be considered as a shape optimization problem. This problem was first considered by Newton, who gave a rather simple variational expression for the aerodynamical resistance of a convex body in a fluid stream, assuming that the competing bodies are radially symmetric, which makes the problem onedimensional. Here are his words (from Principia Mathematica):

If in a rare medium, consisting of equal particles freely disposed at equal distances from each other, a globe and a cylinder described on equal diameter move with equal velocities in the direction of the axis of the cylinder, (then) the resistance of the globe will be half as great as that of the cylinder. ... I 
reckon that this proposition will be not without application in the building of ships.

Under the assumption that the resistance is due to the impact of fluid particles against the body surface, that the particles are supposed all independent (which is quite reasonable if the fluid is rarefied), and that the tangential friction is negligible, by simple geometric considerations we may obtain the following expression of the resistance along the direction of the fluid stream, where we normalize all the physical constants to one:

$$
F(u)=\int_{\Omega} \frac{1}{1+|D u|^{2}} d x .
$$

In the expression above we denoted by $\Omega$ the cross section of the body at the basis level, and by $u(x)$ a function whose graph is the body boundary. The geometrical constraint in the problem consists in requiring that the admissible competing bodies be convex; this is also consistent with the physical assumption that all the fluid particles hit the body at most once. In problem (3) this turns out to be equivalent to assume that $\Omega$ is convex and that $u: \Omega \rightarrow[0,+\infty[$ is concave.

The problem, as considered by Newton, is when $\Omega$ is a disc (of radius $R$ ) and the competing functions are supposed a priori with a radial symmetry. In this case, after integration in polar coordinates, the expression of the resistance takes the form

$$
F(u)=2 \pi \int_{0}^{R} \frac{r}{1+\left|u^{\prime}(r)\right|^{2}} d r
$$

so that the problem of the determination of the optimal radial profile becomes

$$
\min \left\{\int_{0}^{R} \frac{r}{1+\left|u^{\prime}(r)\right|^{2}} d r: u \text { concave, } 0 \leq u \leq M\right\} .
$$

If instead of the functions $u(r)$ we use the functions $v(t)=u^{-1}(M-t)$, the minimization problem (4) can be rewritten in the more traditional form

$$
\min \left\{\int_{0}^{M} \frac{v v^{\prime 3}}{1+v^{\prime 2}} d t: v \text { concave, } v(0)=0, v(M)=R\right\} .
$$

The functional appearing in (5) has to be intended in the sense of $B V$ functions; in fact $v$ is a nondecreasing function, so that $v^{\prime}$ is a nonnegative measure and the precise expression of the functional in (5) is

$$
\int_{0}^{M} \frac{v{v^{\prime}}_{a}^{3}}{1+{v^{\prime}}_{a}^{2}} d t+\int_{[0, M]} v v_{s}^{\prime}=\frac{R^{2}}{2}-\int_{0}^{M} \frac{v v_{a}^{\prime}}{1+v_{a}^{\prime 2}} d t
$$

where $v_{a}^{\prime}$ and $v_{s}^{\prime}$ are respectively the absolutely continuous and singular parts of the measure $v^{\prime}$ with respect to Lebesgue measure. 
It is possible to compute explicitly the solution of the minimization problem (4) by integrating its Euler-Lagrange equation which reads as

$$
r u^{\prime}=C\left(1+u^{\prime 2}\right)^{2} \quad \text { on }\left\{u^{\prime} \neq 0\right\}
$$

for a suitable constant $C<0$. By introducing the function

$$
f(t)=\frac{t}{\left(1+t^{2}\right)^{2}}\left(-\frac{7}{4}+\frac{3}{4} t^{4}+t^{2}-\log t\right) \quad \forall t \geq 1
$$

and the quantities

$$
T=f^{-1}(M / R), \quad r_{0}=\frac{4 R T}{\left(1+T^{2}\right)^{2}},
$$

the solution $u$ can be obtained as

$$
u(r)=M \quad \forall r \in\left[0, r_{0}\right]
$$

and for $r>r_{0}$ its expression is given in parametric form by:

$$
\left\{\begin{array}{l}
r(t)=\frac{r_{0}}{4 t}\left(1+t^{2}\right)^{2} \\
u(t)=M-\frac{r_{0}}{4}\left(-\frac{7}{4}+\frac{3}{4} t^{4}+t^{2}-\log t\right)
\end{array} \quad \forall t \in[1, T] .\right.
$$

We have $\left|u^{\prime}(r)\right|>1$ for all $r>r_{0}$ and $\left|u^{\prime}\left(r_{0}^{+}\right)\right|=1$; moreover, the optimal radial solution can be shown to be unique.

The optimal radial solution of the Newton problem for $M=R$ is shown in figure below.

Coming back to the Newton problem in its general form (not necessarily restricted to radial functions)

$$
\min \{F(u): u \text { concave, } 0 \leq u \leq M\}
$$

where the functional $F$ is given by (3), we notice that the integral functional $F$ above is neither convex nor coercive. Therefore, the usual direct methods of the calculus of variations for obtaining the existence of an optimal solution may fail. However, due to the concavity constraint, the existence of a minimizer $u$ still holds, as it has been proved in [59]. A complete discussion on the problem above can be found in [37] where all the concerning references are quoted. Here we simply recall an interesting necessary condition of optimality (see [119]): it turns out that on every open set $\omega$ where $u$ is of class $C^{2}$ we obtain

$$
\operatorname{det} D^{2} u(x)=0 \quad \forall x \in \omega .
$$

In particular, as it is easy to see, this excludes that in the case $\Omega=B(0, R)$ the solution $u$ be radially symmetric. A profile better than all radial profiles with the same height has been found by Guasoni in [111] and is graphically represented in the figure below. Nevertheless, a complete characterization of the optimal solutions in the case $\Omega=B(0, R)$ is not yet know. 


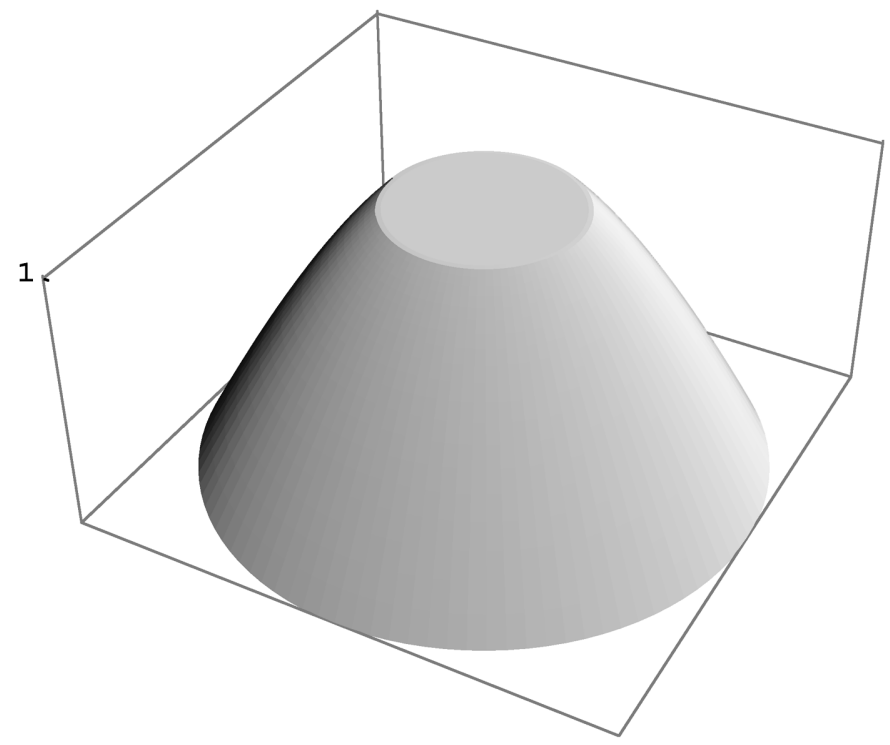

Fig. 1. The optimal radial shape for $M=R$.

\subsection{Optimal Dirichlet regions}

We consider now the model example of a Dirichlet problem over an unknown domain, which has to be optimized according to a given cost functional. Denoting by $f$ a given function, say in $L^{2}\left(\mathbb{R}^{N}\right)$, by $A$ the unknown domain, and by $u_{A}$ the solution of the elliptic equation

$$
-\Delta u=f \text { in } A, \quad u \in H_{0}^{1}(A)
$$

extended by zero outside $A$, the optimization problem we consider is

$$
\min \left\{\int_{Q} j\left(x, u_{A}\right) d x: A \subset Q\right\} .
$$

Here $Q$ is a given bounded domain of $\mathbb{R}^{N}$ and $j(x, s)$ a given integrand.

We refer to the lecture notes [37] for a more complete discussion on this topic and for a wide list of references devoted to this subject; here we want only to summarize the different situations that may occur.

It is well known that in general one should not expect the existence of an optimal solution; for instance, if we take

$$
j(x, s)=|s-a(x)|^{2}
$$

in general, even if the function $a(x)$ is constant, the existence of an optimal solution may fail. However, the existence of an optimal domain occurs in some particular cases that we list below. 


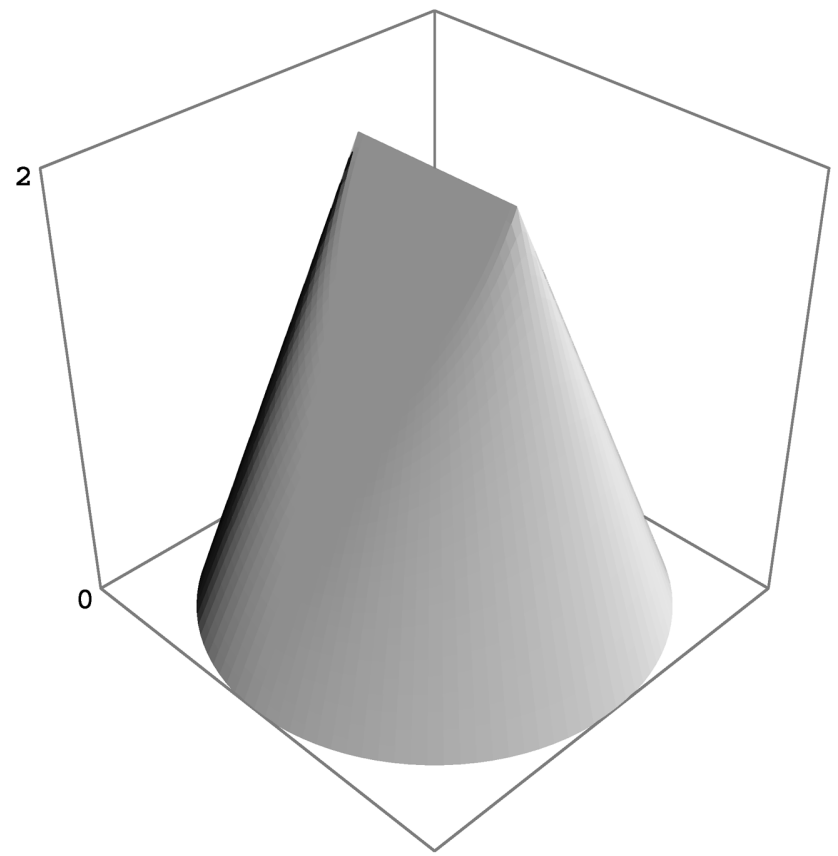

Fig. 2. A nonradial profile better than all radial ones.

i) On the class of admissible domains some rather severe geometrical constraints are imposed. For instance the so called it exterior cone condition is sufficient to imply the existence of an optimal solution. It consists in requiring the existence of a given height $h$ and opening $\omega$ such that for all domains $A$ in the admissible class $\mathcal{A}$ and for all points $x_{0} \in \partial A$ a cone with vertex $x_{0}$, height $h$ and opening $\omega$ is contained in the complement of $A$ (see [48], [49], [50]).

ii) The cost functional fulfills some particular qualitative assumptions. In particular, if a functional $F(A)$ is nonincreasing with respect to the set inclusion (and satisfies a rather mild lower semicontinuity assumption with respect to a $\gamma$-convergence on domains, suitably defined), then the minimization problem

$$
\min \{F(A): A \subset Q, \operatorname{meas}(A) \leq m\}
$$

admits a solution (see [55]).

iii) The problem is of a very special type, involving only the first two eigenvalues of the Laplace operator, where neither geometrical constraints nor monotonicity of the cost are required (see [38]). 
We want to stress that, though quite particular, the previous case ii) covers some interesting situations. For instance cost functionals of the form

$$
F(A)=\int_{Q} j\left(x, u_{A}\right) d x
$$

with $j(x, \cdot)$ nonincreasing, fulfill the required assumptions. Another interesting situation is given by functions of eigenvalues $\lambda_{k}(A)$ of an elliptic operator $L$ with Dirichlet boundary conditions on $\partial A$. In this case, setting $\Lambda(A)=$ $\left(\lambda_{k}\right)_{k \in \mathbb{N}}$, and using the well known fact that the eigenvalues of an elliptic operator are nonincreasing functions of the domain, the functional

$$
F(A)=\Phi(\Lambda(A))
$$

fulfills the required assumptions as soon as the function $\Phi: \mathbb{R}^{\mathbb{N}} \rightarrow[0,+\infty]$ is lower semicontinuous, that is

$$
\left(\Lambda_{n}\right)_{k} \rightarrow(\Lambda)_{k} \quad \forall k \in \mathbb{N} \Rightarrow \Phi(\Lambda) \leq \liminf _{n} \Phi\left(\Lambda_{n}\right)
$$

and nondecreasing, that is

$$
\left(\Lambda_{1}\right)_{k} \leq\left(\Lambda_{2}\right)_{k} \quad \forall k \in \mathbb{N} \quad \Rightarrow \quad \Phi\left(\Lambda_{1}\right) \leq \Phi\left(\Lambda_{2}\right)
$$

\subsection{Optimal mixtures of two conductors}

An interesting question that can be seen in the form of a shape optimization problem is the determination of the optimal distribution of two given conductors (for instance in the thermostatic model, where the state function is the temperature of the system) into a given set. If $\Omega$ denotes a given bounded open subset of $\mathbb{R}^{N}$ (the prescribed container), denoting by $\alpha$ and $\beta$ the conductivities of the two materials, the problem consists in filling $\Omega$ with the two materials in the most performant way according to some given cost functional. The volume of each material can also be prescribed. We denote by $A$ the domain where the conductivity is $\alpha$ and by $a_{A}(x)$ the conductivity coefficient

$$
a_{A}(x)=\alpha 1_{A}(x)+\beta 1_{\Omega \backslash A}(x) .
$$

Then the state equation which associates the control $A$ to the state $u$ (the temperature of the system, once the conductor $\alpha$ fills the domain $A$ ) becomes

$$
\begin{cases}-\operatorname{div}\left(a_{A}(x) D u\right)= & \text { in } \Omega \\ u=0 & \text { on } \partial \Omega,\end{cases}
$$

where $f$ is the (given) source density. We denote by $u_{A}$ the unique solution of (7).

It is well known (see for instance Kohn and Strang [117], Murat and Tartar [138]) that if we take as a cost functional an integral of the form 


$$
\int_{\Omega} j\left(x, 1_{A}, u_{A}, D u_{A}\right) d x
$$

in general an optimal configuration does not exist. However, the addition of a perimeter penalization is enough to imply the existence of classical optimizers. More precisely, we take as a cost the functional

$$
J(u, A)=\int_{\Omega} j\left(x, 1_{A}, u, D u\right) d x+\sigma \operatorname{Per}_{\Omega}(A)
$$

where $\sigma>0$, and the optimal control problem then takes the form

$$
\min \{J(u, A): A \subset \Omega, u \text { solves }(7)\} .
$$

A volume constraint of the form meas $(A)=m$ could also be present. The proof of the existence of an optimal classical solution for problem (8) uses the following result.

Proposition 2.1. For every $n \in \mathbb{N}$ let $a_{n}(x)$ be a $N \times N$ symmetric matrix, with measurable coefficients, such that the uniform ellipticity condition

$$
c_{0}|z|^{2} \leq a_{n}(x) z \cdot z \leq c_{1}|z|^{2} \quad \forall x \in \Omega, \forall z \in \mathbb{R}^{N}
$$

holds with $0<c_{0} \leq c_{1}$ (independent of $n$ ). Given $f \in H^{-1}(\Omega)$ denote by $u_{n}$ the unique solution of the problem

$$
-\operatorname{div}\left(a_{n}(x) D u\right)=f, \quad u_{n} \in H_{0}^{1}(\Omega) .
$$

If $a_{n}(x) \rightarrow a(x)$ a.e. in $\Omega$ then $u_{n} \rightarrow u$ weakly in $H_{0}^{1}(\Omega)$, where $u$ is the solution of (10) with $a_{n}$ replaced by $a$.

Proof. By the uniform ellipticity condition (9) we have

$$
c_{0} \int_{\Omega}\left|D u_{n}\right|^{2} d x \leq \int_{\Omega} f u_{n} d x
$$

and, by the Poincaré inequality we have that $u_{n}$ are bounded in $H_{0}^{1}(\Omega)$ so that a subsequence (still denoted by the same indices) converges weakly in $H_{0}^{1}(\Omega)$ to some $v$. All we have to show is that $v=u$ or equivalently that

$$
-\operatorname{div}(a(x) D v)=f .
$$

This means that for every smooth test function $\phi$ we have

$$
\int_{\Omega} a(x) D v D \phi d x=\langle f, \phi\rangle .
$$

Then it is enough to show that for every smooth test function $\phi$ we have

$$
\lim _{n \rightarrow+\infty} \int_{\Omega} a_{n}(x) D u_{n} D \phi d x=\int_{\Omega} a(x) D v D \phi d x .
$$


This is an immediate consequence of the fact that $\phi$ is smooth, $D u_{n} \rightarrow D v$ weakly in $L^{2}(\Omega)$, and $a_{n} \rightarrow a$ a.e. in $\Omega$ remaining bounded.

Another way to show that (11) holds is to verify that $v$ minimizes the functional

$$
F(w)=\int_{\Omega} a(x) D w D w d x-2\langle f, w\rangle \quad w \in H_{0}^{1}(\Omega) .
$$

Since the function $\alpha(s, z)=s z \cdot z$, defined for $z \in \mathbb{R}^{N}$ and for $s$ symmetric positive definite $N \times N$ matrix, is convex in $z$ and lower semicontinuous in $s$, the functional

$$
\Phi(a, \xi)=\int_{\Omega} a(x) \xi \cdot \xi d x
$$

is sequentially lower semicontinuous with respect to the strong $L^{1}$ convergence on $a$ and the weak $L^{1}$ convergence on $\xi$ (see for instance [52]). Therefore we have

$$
F(v)=\Phi(a, D v)-2\langle f, v\rangle \leq \liminf _{n \rightarrow+\infty} \Phi\left(a_{n}, D u_{n}\right)-2\left\langle f, u_{n}\right\rangle=\liminf _{n \rightarrow+\infty} F\left(u_{n}\right) .
$$

Since $u_{n}$ minimizes the functional $F_{n}$ defined as in (12) with $a$ replaced by $a_{n}$, we also have for every $w \in H_{0}^{1}(\Omega)$

$$
F_{n}\left(u_{n}\right) \leq F_{n}(w)=\int_{\Omega} a_{n}(x) D w D w d x-2\langle f, w\rangle
$$

so that taking the limit as $n \rightarrow+\infty$ and using the convergence $a_{n} \rightarrow a$ we obtain

$$
\liminf _{n \rightarrow+\infty} F_{n}\left(u_{n}\right) \leq \int_{\Omega} a(x) D w D w d x-2\langle f, w\rangle=F(w) .
$$

Thus $F(v) \leq F(w)$ which shows what required.

Remark 2.1. The result of proposition above can be equivalently rephrased in terms of $G$-convergence by saying that for uniformly elliptic operators of the form $-\operatorname{div}(a(x) D u)$ the $G$-convergence is weaker than the $L^{1}$-convergence of coefficients. Analogously, we can say that the functionals

$$
G_{n}(w)=\int_{\Omega} a_{n}(x) D w D w d x
$$

$\Gamma$-converge to the functional $G$ defined in the same way with $a$ in the place of $a_{n}$.

Corollary 2.1. If $A_{n} \rightarrow A$ in $L^{1}(\Omega)$ then $u_{A_{n}} \rightarrow u_{A}$ weakly in $H_{0}^{1}(\Omega)$.

A more careful inspection of the proof of Proposition 2.1 shows that the following stronger result holds. 
Proposition 2.2. Under the same assumptions of Proposition 2.1 the convergence of $u_{n}$ is actually strong in $H_{0}^{1}(\Omega)$.

Proof. In Proposition 2.1 we have already seen that $u_{n} \rightarrow u$ weakly in $H_{0}^{1}(\Omega)$, which gives $D u_{n} \rightarrow D u$ weakly in $L^{2}(\Omega)$. Denoting by $c_{n}(x)$ and $c(x)$ the square root matrices of $a_{n}(x)$ and $a(x)$ respectively, we have that $c_{n} \rightarrow c$ a.e. in $\Omega$ remaining equi-bounded. Then $c_{n}(x) D u_{n}$ converge to $c(x) D u$ weakly in $L^{2}(\Omega)$. Multiplying equation (10) by $u_{n}$ and integrating by parts we obtain

$$
\int_{\Omega} a(x) D u D u d x=\langle f, u\rangle=\lim _{n \rightarrow+\infty}\left\langle f, u_{n}\right\rangle=\lim _{n \rightarrow+\infty} \int_{\Omega} a_{n}(x) D u_{n} D u_{n} d x .
$$

This implies that

$$
c_{n}(x) D u_{n} \rightarrow c(x) D u \quad \text { strongly in } L^{2}(\Omega) .
$$

Multiplying now by $\left(c_{n}(x)\right)^{-1}$ we finally obtain the strong convergence of $D u_{n}$ to $D u$ in $L^{2}(\Omega)$.

We are now in a position to obtain an existence result for the optimization problem (8). On the function $j$ we only assume that it is nonnegative, Borel measurable, and such that $j(x, s, z, w)$ is lower semicontinuous in $(s, z, w)$ for a.e. $x \in \Omega$.

Theorem 2.1. Under the assumptions above the minimum problem (8) admits at least a solution.

Proof. Let $\left(A_{n}\right)$ be a minimizing sequence; then $\operatorname{Per}_{\Omega}\left(A_{n}\right)$ are bounded, so that, up to extracting subsequences, we may assume $\left(A_{n}\right)$ is strongly convergent in the $L^{1}$ sense to some set $A \subset \Omega$. We claim that $A$ is a solution of problem (8). Let us denote by $u_{n}$ a solution of problem (7) associated to $A_{n}$; by Proposition $2.2\left(u_{n}\right)$ converges strongly in $H_{0}^{1}(\Omega)$ to some $u \in H_{0}^{1}(\Omega)$. Then by the lower semicontinuity of the perimeter and by Fatou's lemma we have

$$
J(u, A) \leq \liminf _{n \rightarrow+\infty} J\left(u_{n}, A_{n}\right)
$$

which proves the optimality of $A$.

Remark 2.2. The existence result above still holds, with the same proof, when volume constraints of the form meas $(A)=m$ are present. Indeed this constraint passes to the limit when $A_{n} \rightarrow A$ strongly in $L^{1}(\Omega)$.

The existence result above shows the existence of a classical solution for the optimization problem (8). This solution is simply a set with finite perimeter and additional assumptions have to be made in order to prove further 
regularity. For instance in [8] Ambrosio and Buttazzo considered the similar problem

$$
\min \left\{E(u, A)+\sigma \operatorname{Per}_{\Omega}(A): u \in H_{0}^{1}(\Omega), A \subset \Omega\right\}
$$

where $\sigma>0$ and

$$
E(u, A)=\int_{\Omega}\left[a_{A}(x)|D u|^{2}+1_{A}(x) g_{1}(x, u)+1_{\Omega \backslash A} g_{2}(x, u)\right] d x .
$$

They showed that every solution $A$ is actually an open set provided $g_{1}$ and $g_{2}$ are Borel measurable and satisfy the inequalities

$$
g_{i}(x, s) \geq \gamma(x)-k|s|^{2} \quad i=1,2
$$

where $\gamma \in L^{1}(\Omega)$ and $k<\alpha \lambda_{1}$, being $\lambda_{1}$ the first eigenvalue of $-\Delta$ on $\Omega$.

\section{Mass optimization problems}

In this section we present an optimization problem that we call mass optimization problem; it plays a central role in many questions in Applied Mathematics and Engineering. It consists in finding the elastic structure (seen as a distribution of a given amount of elastic material) that, for a given system $f$ of loads and for a given total mass, gives the best resistance in terms of minimal compliance. The unknown mass distribution is then a nonnegative measure which may vary in the class of admissible choices, with total mass prescribed, and support possibly constrained in a given design region.

In order to take into account also forces which may concentrate on lower dimensional sets we consider a force field $f \in \mathcal{M}\left(\mathbb{R}^{N} ; \mathbb{R}^{N}\right)$, the class of all $\mathbb{R}^{N}$-valued measures on $\mathbb{R}^{N}$ with finite total variation and with compact support. The class of smooth displacements we consider is the Schwartz space $\mathcal{D}\left(\mathbb{R}^{N} ; \mathbb{R}^{N}\right)$ of $C^{\infty}$ functions with compact support; similarly, the notation $\mathcal{D}^{\prime}\left(\mathbb{R}^{N} ; \mathbb{R}^{N}\right)$ stands for the space of vector valued distributions and, for a given nonnegative measure $\mu, L_{\mu}^{p}\left(\mathbb{R}^{N} ; \mathbb{R}^{d}\right)$ denotes the space of $p$-integrable functions with respect to $\mu$ with values in $\mathbb{R}^{d}$.

For every $N \times N$ matrix $z$ we denote by $z^{\text {sym }}$ the symmetric part of $z$ and by $e(u)$ the strain tensor $(D u)^{s y m}$; in this way, for a given smooth displacement $u: \mathbb{R}^{N} \rightarrow \mathbb{R}^{N}$ we denote by $j(D u)=j(e(u))$ the stored elastic energy density associated to $u$, where

$$
j(z)=\beta\left|z^{s y m}\right|^{2}+\frac{\alpha}{2}\left|\operatorname{tr}\left(z^{s y m}\right)\right|^{2}
$$

being $\alpha$ and $\beta$ are the so called Lamé constants. This is the case when the material to distribute is a homogeneous isotropic linearly elastic material; the same analysis holds if more generally we assume:

i) $j$ is convex and positively $p$-homogeneous, with $p>1$; 
ii) $j(z)=j\left(z^{s y m}\right)$;

iii) there exist two positive constants $\alpha_{1}$ and $\alpha_{2}$ such that

$$
\alpha_{1}\left|z^{s y m}\right|^{p} \leq j(z) \leq \alpha_{2}\left|z^{s y m}\right|^{p} \quad \forall z \in \mathbb{R}^{N \times N} .
$$

For a given mass distribution $\mu$ the stored elastic energy of a smooth displacement $u \in \mathcal{D}\left(\mathbb{R}^{N} ; \mathbb{R}^{N}\right)$ is given by

$$
J(\mu, u)=\int j(D u) d \mu
$$

so that the total energy associated to $\mu$ and relative to a smooth displacement $u$ is

$$
E(\mu, u)=J(\mu, u)-\langle f, u\rangle
$$

where $\langle f, u\rangle$ represents the work of the force field $f$.

In order to take into account possibly prescribed Dirichlet boundary conditions, we consider a closed subset $\Sigma$ of $\mathbb{R}^{N}$ (when $\Sigma=\emptyset$ the problem is called of pure traction type) and we impose the admissible displacements vanish on $\Sigma$. Thus we may now define the energy of a measure $\mu$ as the infimum

$$
\mathcal{E}(\mu)=\inf \left\{E(\mu, u): u \in \mathcal{D}\left(\mathbb{R}^{N} ; \mathbb{R}^{N}\right), u=0 \text { on } \Sigma\right\}
$$

and the compliance $\mathcal{C}(\mu)$ is then defined as

$$
\mathcal{C}(\mu)=-\mathcal{E}(\mu)
$$

The optimization problem we want to consider is then

$$
\min \left\{\mathcal{C}(\mu): \mu \in \mathcal{M}^{+}\left(\mathbb{R}^{N}\right), \int d \mu=m, \operatorname{spt} \mu \subset K\right\}
$$

where the total mass constraint $\int d \mu=m$ is present, and where a design region constraint is also possible, which turns out to give a closed subset $K$ of $\mathbb{R}^{N}$ and to limit the analysis only to mass distributions which vanish outside $K$. We assume that $K$ is the closure $\bar{\Omega}$ of a smooth connected bounded open subset $\Omega$ of $\mathbb{R}^{N}$. It should also be noticed that the problem above is a variational model which describes the behaviour of light structures, where the force due to their own weight is neglected.

Since for a fixed smooth admissible displacement $u$ the mapping $\mu \mapsto$ $E(\mu, u)$ is affine and continuous for the weak* convergence, the functional $\mathcal{C}(\mu)$ turns out to be convex and lower semicontinuous. Therefore, by direct methods of the calculus of variations we obtain the following existence result.

Theorem 3.1. The mass optimization problem (15) admits at least a solution. 
Remark 3.1. The same formulation can be used for the case of scalar state functions $u$; in this case the prototype of the function $j(z)$ is the Dirichlet energy density

$$
j(z)=\frac{1}{2}|z|^{2}
$$

and the optimization problem (15) describes the optimal distribution of a given amount of conducting (for instance in the thermostatic model) material, being $f$ the heat sources density.

Remark 3.2. We want to stress that we may have $\mathcal{C}(\mu)=+\infty$ for some measures $\mu$; this happens for instance in the case when the force field $f$ concentrates on sets of dimension smaller than $n-1$ and the mass distribution $\mu$ is the Lebesgue measure. However, these "singular" measures $\mu$ which have infinite compliance are ruled out from our discussion because we look for the minimization of the compliance functional $\mathcal{C}(\mu)$.

By standard duality arguments (see for instance [98]) we may rewrite the compliance $\mathcal{C}(\mu)$ in the form

$$
\begin{aligned}
\mathcal{C}(\mu)=\inf \left\{\int j^{*}(\sigma) d \mu: \sigma \in L_{\mu}^{p^{\prime}}\left(\mathbb{R}^{N} ; \mathbb{R}^{N \times N}\right)\right. & \\
& \left.-\operatorname{div}(\sigma \mu)=f \text { in } \mathcal{D}^{\prime}\left(\mathbb{R}^{N} \backslash \Sigma ; \mathbb{R}^{N}\right)\right\}
\end{aligned}
$$

and the infimum in (16) is actually a minimum as soon as $\mathcal{C}(\mu)$ is finite.

In order to characterize the optimal solutions $\mu_{\text {opt }}$ of problem (15) by means of necessary and sufficient conditions of optimality it is convenient to introduce the quantity

$$
I(f, \Sigma, \Omega)=\sup \{\langle f, u\rangle: u=0 \text { on } \Sigma, j(D u) \leq 1 / p \text { on } \bar{\Omega}\} .
$$

The following result holds (we refer to [23] for the proof).

Proposition 3.1. The mass optimization problem (15) is nontrivial, in the sense that the compliance functional $\mathcal{C}$ is not identically $+\infty$, provided $I(f, \Sigma$, $\Omega)$ is finite. Moreover, for every nonnegative measure $\mu$ with $\int d \mu=m$ and $\operatorname{spt} \mu \subset \bar{\Omega}$ we have

$$
\mathcal{C}(\mu) \geq \frac{(I(f, \Sigma, K))^{p^{\prime}}}{p^{\prime} m^{1 /(p-1)}} .
$$

Finally, there exists a nonnegative measure $\mu$ with $\int d \mu=m$ and $\operatorname{spt} \mu \subset K$ such that

$$
\mathcal{C}(\mu) \leq \frac{(I(f, \Sigma, \Omega))^{p^{\prime}}}{p^{\prime} m^{1 /(p-1)}} .
$$


The optimal mass distribution $\mu_{\text {opt }}$ then verifies the equality

$$
\mathcal{C}\left(\mu_{\text {opt }}\right)=\frac{(I(f, \Sigma, \Omega))^{p^{\prime}}}{p^{\prime} m^{1 /(p-1)}} .
$$

The quantity $I(f, \Sigma, \Omega)$ can also be related to the dual problem (16) by introducing the 1-homogeneous function $\rho(z)$ as

$$
\rho(z)=\inf \{t>0: j(z / t) \leq 1 / p\}
$$

so that we have

$$
j(z)=\frac{1}{p}(\rho(z))^{p} .
$$

The polar function associated to $\rho$ is given by:

$$
\rho^{0}(z)=\sup \{z: \xi: \rho(\xi) \leq 1\}
$$

and we obtain

$$
j^{*}(z)=\frac{1}{p^{\prime}}\left(\rho^{0}(z)\right)^{p^{\prime}} \quad \forall z \in \mathbb{R}^{N \times N} .
$$

In this way we have the equality

$$
\begin{aligned}
I(f, \Sigma, \Omega)=\inf \left\{\int \rho^{0}(\lambda): \lambda\right. & \in \mathcal{M}\left(\mathbb{R}^{N} ; \mathbb{R}^{N \times N}\right), \\
\operatorname{spt} \lambda & \left.\subset \bar{\Omega},-\operatorname{div} \lambda=f \operatorname{in} \mathcal{D}^{\prime}\left(\mathbb{R}^{N} \backslash \Sigma ; \mathbb{R}^{N}\right)\right\},
\end{aligned}
$$

where the integral is intended in the sense of convex functionals on measures, and the following result holds (see [23]).

Proposition 3.2. If $\mu$ is a solution of the mass optimization problem (15) then one has

$$
\begin{aligned}
I(f, \Sigma, \Omega)=\min \left\{\int \rho^{0}(\sigma) d \mu:\right. & \sigma \in L_{\mu}^{1}\left(\mathbb{R}^{N} ; \mathbb{R}^{N \times N}\right), \\
& \left.-\operatorname{div}(\sigma \mu)=f \text { in } \mathcal{D}^{\prime}\left(\mathbb{R}^{N} \backslash \Sigma ; \mathbb{R}^{N}\right)\right\}
\end{aligned}
$$

and every optimal $\sigma$ in (20) verifies

$$
\rho^{0}(\sigma)=\frac{I(f, \Sigma, \Omega)}{m} \quad \mu \text {-almost everywhere. }
$$

Conversely, if $\lambda$ is a solution of (19), then the nonnegative measure

$$
\mu:=\frac{m}{I(f, \mathcal{U}, \Omega)} \rho^{0}(\lambda)
$$

is optimal for (15). 
Remark 3.3. It is interesting to notice that by Proposition 3.1 the optimal mass distributions for problem (15) can be deduced from solutions of problem (19), hence they do not depend on the growth exponent $p$ of the energy density $j$ but only on the convex level set $\left\{z \in \mathbb{R}^{N \times N}: \rho(z) \leq 1\right\}$. Moreover, when $\mu$ is an optimal mass distribution, by (21) the associated stress density $j^{*}(\sigma)=\frac{1}{p^{\prime}}\left(\rho^{0}(\sigma)\right)^{p^{\prime}}$ is constant.

The optimal mass distribution problem (15) can be equivalently rephrased in terms of a PDE that we call Monge-Kantorovich equation. In order to deduce this equation from the mass optimization problem, it is convenient to introduce the class $\operatorname{Lip}_{1, \rho}(\Omega, \Sigma)$ as the closure, in $C\left(\bar{\Omega} ; \mathbb{R}^{N}\right)$, of the set $\left\{u \in \mathcal{D}\left(\mathbb{R}^{N} ; \mathbb{R}^{N}\right): \rho(D u) \leq 1\right.$ on $\Omega, u=0$ on $\left.\Sigma\right\}$. We notice that when $\rho(z) \geq|z|$ then every function in $\operatorname{Lip}_{1, \rho}(\Omega)$ is locally Lipschitz continuous on $\Omega$; on the other hand, if $\rho(z)=\left|z^{\text {sym }}\right|$ it is known that this is no more true, due to the lack of Korn inequality for $p=+\infty$ (see for instance [94]).

We can now define the relaxed formulation of problem (17) as

$$
\sup \left\{\langle f, u\rangle: u \in \operatorname{Lip}_{1, \rho}(\Omega, \Sigma)\right\}
$$

and the finite dimensional linear space of all rigid displacements vanishing on $\Sigma$

$$
\mathcal{R}_{\Sigma}=\left\{u(x)=A x+b: b \in \mathbb{R}^{N}, A \in \mathbb{R}_{\text {skew }}^{N \times N}, u=0 \text { on } \Sigma\right\} .
$$

Proposition 3.3. The supremum in problem (17) is finite if and only if

$$
\langle f, u\rangle=0 \quad \forall u \in \mathcal{R}_{\Sigma} .
$$

In this case, problem (22) admits a solution and

$$
\sup (17)=\max (22) \text {. }
$$

Remark 3.4. In the scalar case, with $\rho(z)=|z|$, it is easy to see that the class $\mathcal{R}_{\Sigma}$ reduces to the function identically zero when $\Sigma \neq \emptyset$ and to the family of constant functions when $\Sigma=\emptyset$. Therefore, in the scalar case the quantity $I(f, \Sigma, \Omega)$ is always finite whenever $\Sigma \neq \emptyset$ or, if $\Sigma=\emptyset$, provided the source $f$ has zero average.

In order to well define the optimality conditions for problem (15) and to deduce the Monge-Kantorovich PDE, we need to introduce the function space of displacements of finite energy related to a general measure $\mu$. We refer to [27] and [28] for a complete presentation of the tools we shall use; in particular for the notion of tangent bundle of a measure $\mu$, generalizing the classical one of $k$-dimensional manifolds $S$, which correspond in this framework to the measures of the form $\mu=\mathcal{H}^{k}\llcorner S$.

Given a measure $\mu$ and an open subset $U$ of $\mathbb{R}^{N}$ we define the space of admissible stresses 


$$
X_{\mu}^{p^{\prime}}\left(U ; \mathbb{R}_{s y m}^{N \times N}\right)=\left\{\sigma \in L_{\mu}^{p^{\prime}}\left(U ; \mathbb{R}_{s y m}^{N \times N}\right): \operatorname{div}(\sigma \mu) \in \mathcal{M}\left(\mathbb{R}^{N} ; \mathbb{R}^{N}\right)\right\}
$$

and the tangent set of matrices

$$
M_{\mu}(x)=\mu-e s s \bigcup\left\{\sigma(x): \sigma \in X_{\mu}^{p^{\prime}}\left(U ; \mathbb{R}_{s y m}^{N \times N}\right)\right\},
$$

where $\mu$-ess stands for the $\mu$ essential union. If $P_{\mu}(x)$ denotes the orthogonal projector on $M_{\mu}(x)$ with respect to the usual scalar product on matrices, for every function $u \in \mathcal{D}\left(U ; \mathbb{R}^{N}\right)$ we may then define the tangential strain $e_{\mu}(u)$ as

$$
e_{\mu}(u)(x)=P_{\mu}(x) D u(x) .
$$

It is possible to show that the linear operator

$$
u \in \mathcal{D}\left(U ; \mathbb{R}^{N}\right) \mapsto e_{\mu}(u) \in L_{\mu}^{p}\left(U ; \mathbb{R}_{\text {sym }}^{N \times N}\right)
$$

is closable as an operator from $C\left(\bar{U} ; \mathbb{R}^{N}\right)$ into $L_{\mu}^{p}\left(U ; \mathbb{R}_{\text {sym }}^{N \times N}\right)$, and we still denote by $e_{\mu}$ the closed operator from $C\left(\bar{U} ; \mathbb{R}^{N}\right)$ into $L_{\mu}^{p}\left(U ; \mathbb{R}_{s y m}^{N \times N}\right)$ which extends the tangential strain.

Now we can define the Banach space of all finite energy displacements $\mathcal{D}_{0, \mu}^{1, p}(U)$ as the domain of the operator $e_{\mu}$ endowed with the norm

$$
\|u\|_{\mathcal{D}_{0, \mu}^{1, p}(U)}=\|u\|_{C(\bar{U})}+\left\|e_{\mu}(u)\right\|_{L_{\mu}^{p}(U)} .
$$

It is then possible to obtain the relaxed form of the stored energy functional $J(\mu, u)$

$$
\begin{aligned}
\bar{J}(\mu, u) & =\inf \left\{\liminf _{h \rightarrow+\infty} J\left(\mu, u_{h}\right): u_{h} \rightarrow u \text { uniformly, } u_{h} \in \mathcal{D}\left(U ; \mathbb{R}^{N}\right)\right\}= \\
& = \begin{cases}\int_{U} j_{\mu}\left(x, e_{\mu}(u)\right) d \mu & \text { if } u \in \mathcal{D}_{0, \mu}^{1, p}(U) \\
+\infty & \text { otherwise. }\end{cases}
\end{aligned}
$$

where

$$
j_{\mu}(x, z)=\inf \left\{j(z+\xi): \xi \in\left(M_{\mu}(x)\right)^{\perp}\right\} .
$$

This allows us to prove that for every nonnegative measure $\mu$ in $\bar{\Omega}$ and for every admissible displacement $u \in \operatorname{Lip}_{1, \rho}(\Omega, \Sigma)$ we have that $u \in \mathcal{D}_{0, \mu}^{1, p}\left(\mathbb{R}^{N} \backslash \Sigma\right)$ and $j_{\mu}\left(x, e_{\mu}(u)\right) \leq 1 / p \mu$-a.e. on $\mathbb{R}^{N} \backslash \Sigma$.

The scalar case is slightly simpler; indeed we define

$$
X_{\mu}^{p^{\prime}}\left(U ; \mathbb{R}^{N}\right)=\left\{\sigma \in L_{\mu}^{p^{\prime}}\left(U ; \mathbb{R}^{N}\right): \operatorname{div}(\sigma \mu) \in \mathcal{M}\left(\mathbb{R}^{N} ; \mathbb{R}^{N}\right)\right\}
$$

and the tangent space $T_{\mu}(x)$ for $\mu$-a.e. $x$ as

$$
T_{\mu}(x)=\mu-\operatorname{ess} \bigcup\left\{\sigma(x): \sigma \in X_{\mu}^{p^{\prime}}\left(U ; \mathbb{R}^{N}\right)\right\} .
$$


The orthogonal projector $P_{\mu}(x)$ on $T_{\mu}(x)$ and the tangential gradient $D_{\mu} u$ are defined similarly as above, as well as the relaxed energy density which becomes

$$
j_{\mu}(x, z)=\inf \left\{j(z+\xi): \xi \in\left(T_{\mu}(x)\right)^{\perp}\right\} .
$$

Note that in the case $j(z)=|z|^{2} / 2$ we obtain $j_{\mu}(x, z)=\left|P_{\mu}(x) z\right|^{2} / 2$. Moreover, in this case $\rho(z)=|z|$ and we have

$$
\operatorname{Lip}_{1, \rho}(\Omega ; \Sigma)=\left\{u \in W^{1, \infty}(\Omega): u=0 \text { on } \Sigma,|D u| \leq 1 \text { a.e. on } \Omega\right\} \text {. }
$$

We are now in a position to introduce the Monge-Kantorovich equation that can be written as:

$$
\left\{\begin{array}{l}
-\operatorname{div}(\sigma \mu)=f \quad \text { on } \mathbb{R}^{N} \backslash \Sigma \\
\sigma \in \partial j_{\mu}\left(x, e_{\mu}(u)\right) \quad \mu \text {-a.e. on } \mathbb{R}^{N} \\
u \in \operatorname{Lip}_{1, \rho}(\Omega, \Sigma) \\
j_{\mu}\left(x, e_{\mu}(u)\right)=1 / p \mu \text {-a.e. on } \mathbb{R}^{N} \\
\mu(\Sigma)=0 .
\end{array}\right.
$$

where $\partial j_{\mu}(x, \cdot)$ denotes the subdifferential of the convex function $j_{\mu}(x, \cdot)$. The link between the mass optimization problem (15) and the Monge-Kantorovich equation above has been investigated in [23]; we summarize here the result.

Theorem 3.2. If $\mu$ solves the mass optimization problem (15) and $u$ and $\sigma$ are solutions of problems (22) and (20) respectively, then the triple ( $u, m \sigma / I$, $I \mu / m)$ solves the Monge-Kantorovich equation (25) with $I=I(f, \Sigma, \Omega)$. Vice versa, if the triple $(u, \sigma, \mu)$ solves the Monge-Kantorovich equation (25), then $u$ is a solution of problem (22) and the measure $m \mu / I$ is a solution of the mass optimization problem (15). Moreover, $I \sigma / m$ is a solution of the stress problem (16), and $(I / m)^{1 /(p-1)} u$ is a solution of the relaxed displacement problem

$$
\min \left\{\bar{J}(\mu, v)-\langle f, v\rangle: v \in \mathcal{D}_{0, \mu}^{1, p}\left(\mathbb{R}^{N} \backslash \Sigma\right)\right\},
$$

both related to the measure $m \mu / I$.

The scalar case is again simpler; indeed, by also taking $\rho(z)=|z|$ the Monge-Kantorovich equation (25) becomes

$$
\left\{\begin{array}{l}
-\operatorname{div}\left(\mu D_{\mu} u\right)=f \quad \text { on } \mathbb{R}^{N} \backslash \Sigma \\
u \in W^{1, \infty}(\Omega), u=0 \text { on } \Sigma,|D u| \leq 1 \text { a.e. on } \Omega \\
\left|D_{\mu} u\right|=1 \quad \mu \text {-a.e. on } \mathbb{R}^{N} \\
\mu(\Sigma)=0 .
\end{array}\right.
$$

\section{Optimal transportation problems}

In this section we introduce the optimal mass transportation problem in his strong form (Monge problem) and in his weak or relaxed form (Kantorovich 
problem). In Section 5 we will enlight some full or partial equivalences with the mass optimization problems introduced in the previous section.

This equivalence will permit to give in the scalar case an explicit formula for the optimal mass and to deduce some regularity results as well as to allow some useful variational approximations of the mass optimization problem.

\subsection{The optimal mass transportation problem: Monge and Kantorovich formulations}

In order to have a general framework, in this section we will work in a locally compact metric space $(X, d)$. This will make more clear various notions and will permit an unified treatment of different situations when studying the relationships with the mass optimization problems. The problem was originally formulated by Monge in 1781 (see [35]) in the Euclidean space. Using a modern terminology we will use measures where Monge was generically speaking of mass densities. This also permits a larger flexibility of the model.

Let $f^{+}$and $f^{-}$be two probability measures on $X$; a transport map of $f^{+}$ on $f^{-}$is an element of the set

$$
T\left(f^{+}, f^{-}\right):=\left\{\varphi: X \rightarrow X \text { s.t. } \varphi \text { is measurable and } \varphi_{\sharp} f^{+}=f^{-}\right\}
$$

where $\varphi_{\sharp} f^{+}$denote the push forward of $f^{+} \operatorname{through} \varphi$ i.e. the measure on $X$ defined as follows:

$$
\varphi_{\sharp} f^{+}(B):=f^{+}\left(\varphi^{-1}(B)\right) \quad \text { for all Borel subsets } B \text { of } X .
$$

A general (justified by physical or economical applications) cost of a transport map in $T\left(f^{+}, f^{-}\right)$is given by

$$
J(\varphi)=\int_{X} \psi\left(d(x, \varphi(x)) d f^{+}(x) .\right.
$$

The quantity $\psi\left(d(x, \varphi(x)) d f^{+}(x)\right.$ represents the cost of transportation for mass unity. Then $\psi: \mathbb{R}^{+} \rightarrow \mathbb{R}^{+}$is a positive increasing function. In the original formulation Monge considered $\psi(t)=t$, while in economical applications it is reasonable to expect that $\psi$ is concave (see for instance [106]); on the other hand, strictly convex functions $\psi$ are used to study certain classes of differential equations (see for instance [12], [140], [154]).

Then a general formulation of the Monge problem is:

$$
\min \left\{\int_{X} \psi\left(d(x, \varphi(x)) d f^{+}(x): \varphi \in T\left(f^{+}, f^{-}\right)\right\} .\right.
$$

Remark 4.1. It may happen that the class $T\left(f^{+}, f^{-}\right)$is empty; this occurs for instance when $f^{-}=\frac{1}{2}\left(\delta_{x_{0}}+\delta_{x_{1}}\right)$ and $f^{+}=\delta_{x_{2}}$ in a space $X$ which contains at least 3 points. Furthermore, even if $T\left(f^{+}, f^{-}\right) \neq \emptyset$, in general the minimum in (30) is not achieved as shown in the next example. 
Example 4.1. Denote by $I$ the interval $[0,1]$ and consider as $X$ the Euclidean plane $\mathbb{R}^{2}, f^{+}=\mathcal{H}^{1}\left\llcorner(\{0\} \times I)\right.$ and $f^{-}=\frac{1}{2} \mathcal{H}^{1}\left\llcorner(\{1\} \times I)+\frac{1}{2} \mathcal{H}^{1}\llcorner(\{-1\} \times I)\right.$. In this case it is easy to see that the infimum in problem (30) is 1 but for each transport map $\varphi$ we have $|x-\varphi(x)| \geq 1$ for $f^{+}$a.e. $x$ and there is a set of positive $f^{+}$-measure for which $|x-\varphi(x)|>1$.

The behaviour of minimizing sequences of transport maps in the previous example shows also that the class of transport maps is not closed with respect to a topology sufficiently strong to deal with the nonlinearity of functional (29). Then it is natural to think to a possible relaxed formulation of the problem. The relaxed formulation we are introducing is due to Kantorovich and it consists in embedding the class of transport maps in the larger class of the so called transport plans which will be introduced below.

A transport plan of $f^{+}$to $f^{-}$is a probability measure $\gamma \in \mathcal{M}(X \times X)$ which belongs to the set:

$$
P\left(f^{+}, f^{-}\right)=\left\{\gamma \in \operatorname{Prob}(X \times X): \pi_{\sharp}^{1} \gamma=f^{+}, \pi_{\sharp}^{2} \gamma=f^{-}\right\},
$$

where for all $(x, y) \in X \times X, \pi^{1}(x, y)=x$ and $\pi^{2}(x, y)=y$ are the projections on the first and second factors of $X \times X$. It is easy to check that (denoting by $i d$ the identity map) $(i d \otimes \varphi)_{\sharp} f^{+} \in P\left(f^{+}, f^{-}\right)$if and only if $\varphi \in T\left(f^{+}, f^{-}\right)$. The reader familiar with the Young measures will recognize in the transport plan associated to a transport map $\varphi$ the measure associated to the graph of $\varphi$. As the embedding of $T\left(f^{+}, f^{-}\right)$in $P\left(f^{+}, f^{-}\right)$is given by $\varphi \mapsto(i d \otimes \varphi)_{\sharp} f^{+}$ the natural extension of the cost functional is given by

$$
J(\gamma)=\int_{X \times X} \psi(d(x, y) d \gamma(x, y),
$$

so that Monge problem (30) relaxes to the Kantorovich problem:

$$
\min \left\{\int_{X \times X} \psi(d(x, y)) d \gamma(x, y): \gamma \in P\left(f^{+}, f^{-}\right)\right\} .
$$

Remark 4.2. The class $P\left(f^{+}, f^{-}\right)$is never empty as it always contains $f^{+} \otimes f^{-}$. Moreover $P\left(f^{+}, f^{-}\right)$is closed with respect to the tight convergence on the space of finite measures. This implies that problem (33) admits a minimizer whenever the function $\psi$ is lower semicontinuous.

Example 4.2. Let $f^{+}$and $f^{-}$be the measures of Example 4.1, then it is easy to see that the only optimal transport plan is given by the probability measure on $\mathbb{R}^{4}$

$$
\gamma=f^{+} \otimes f^{-}
$$

which turns out to verify for every test function $\theta(x, y, z, w)$ on $\mathbb{R}^{4}$

$$
\langle\gamma, \theta\rangle=\frac{1}{2} \int_{0}^{1} \int_{0}^{1}[\theta(0, y, 1, w)+\theta(0, y,-1, w)] d y d w .
$$

Therefore again we conclude that the minimum cannot be achieved on the set of tranport maps. 
We go now back to consider a bounded and connected open subset $\Omega$ of $\mathbb{R}^{N}$ and a closed subset $\Sigma$ of $\bar{\Omega}$. We want to define a semi-distance on $\bar{\Omega}$ that only allows paths in $\bar{\Omega}$ and that does not count the lenght of paths along $\Sigma$. We start with the case when $\Sigma$ is empty: in this case we simply define $d_{\Omega}$ as the geodesic distance in $\bar{\Omega}$, that is

$$
d_{\Omega}(x, y)=\inf \left\{\int_{0}^{1}\left|\xi^{\prime}(t)\right| d t: \xi(0)=x, \xi(1)=y, \xi(t) \in \bar{\Omega}\right\} .
$$

If $\Sigma \neq \emptyset$ we set

$$
d_{\Omega, \Sigma}(x, y)=\min \left\{d_{\Omega}(x, y), d_{\Omega}(x, \Sigma)+d_{\Omega}(y, \Sigma)\right\}
$$

where we denoted, as usual, by $d_{\Omega}(x, \Sigma)$ the $d_{\Omega}$-distance of the point $x$ from the closed set $\Sigma$.

With the notation above the Kantorovich problem, for the choice $\psi(t)=t$, can be written as:

$$
\min \left\{\int_{\Omega \times \Omega} d_{\Omega, \Sigma}(x, y) d \gamma(x, y): \gamma \in P\left(f^{+}, f^{-}\right)\right\} .
$$

By denoting by $\operatorname{Lip}_{1}\left(\Omega, d_{\Omega, \Sigma}\right)$ the class of functions defined in $\Omega$ that are 1-Lipschitz continuous with respect to the distance $d_{\Omega, \Sigma}$ one may obtain a dual formulation of problem (35) in the form (see [23]):

$$
I(f, \Sigma, \Omega):=\max \left\{\langle f, u\rangle: u \in \operatorname{Lip}_{1}\left(\Omega, d_{\Omega, \Sigma}\right), u=0 \text { on } \Sigma\right\} .
$$

\subsection{The PDE formulation of the mass transportation problem}

A system of partial differential equations can be associated to the MongeKantorovich problem (see for instance [23], [99], [100]). In a simplified version (i.e. $\Omega$ convex and bounded, $\Sigma$ empty and $f^{+}$and $f^{-}$regular functions with separated supports) this system was first used to prove the existence of an optimal transport by Evans and Gangbo in [100] and it can be written as follows:

$$
\begin{cases}-\operatorname{div}(a(x) D u(x))=f & \text { in } \Omega \\ u \in \operatorname{Lip}_{1}(\Omega) & a(x) \text {-a.e. }\end{cases}
$$

The geometrical informations about the directions in which the mass has to be transported in order to minimize the cost are contained in the gradient of $u$, while the density of the transport rays is contained in the coefficient $a(x)$.

The interest of this system in the mass optimization theory is due to the fact that it can be used to characterize the solutions of problem (15). We will show more in the next section. 


\section{Relationships between optimal mass and optimal transportation}

We begin to observe that as $j$ is 2 -homogeneous it can be written as $j(z)=$ $\frac{1}{2}(\rho(z))^{2}$ where $\rho$ is positive, convex and 1-homogeneous. Then, as already done in (22), we consider the quantity

$$
I(f, \Sigma, \Omega)=\max \left\{\langle f, u\rangle: u \in \operatorname{Lip}_{1, \rho}(\Omega, \Sigma)\right\} .
$$

Remark 5.1. In the scalar case (with $\Omega$ convex and $\Sigma=\emptyset$, otherwise the same modifications seen above apply, see [23] for a general discussion) the interpretation of (38) in terms of transportation problem is related to the distance defined by

$$
d_{\rho}(x, y)=\inf \left\{\int_{0}^{1} \rho^{0}\left(\xi^{\prime}(t)\right) d t: \xi(0)=x, \xi(1)=y\right\}
$$

where $\rho^{0}(z):=\sup \{w \cdot z: \rho(w) \leq 1\}$.

Using the duality between continuous functions and measures one obtains the identity:

$$
I(f, \Sigma, \Omega)=\inf \left\{\int \rho^{0}(\lambda): \lambda \in \mathcal{M}(\bar{\Omega}),-\operatorname{div} \lambda=f \text { in } \mathbb{R}^{N} \backslash \Sigma\right\} .
$$

Here the integral $\int \rho^{0}(\lambda)$ is intended in the sense of convex functions of a measure; more precisely, if $\theta$ is a convex function and $\lambda$ is a vector measure, we set

$$
\int \theta(\lambda):=\int \theta\left(\lambda^{a}\right) d x+\int \theta^{\infty}\left(\frac{d \lambda^{s}}{d\left|\lambda^{s}\right|}\right) d \mid \lambda^{s}
$$

where $\theta^{\infty}$ is the recession function of $\theta$ and $\lambda=\lambda^{a} d x+\lambda^{s}$ is the decomposition of $\lambda$ into its absolutely continuous part $\lambda^{a}$ and singular part $\lambda^{s}$. Finally we remark that if $\theta$ is 1 -homogeneous (as for instance in the case of $\theta=\rho^{0}$ ) then $\theta$ coincides with $\theta^{\infty}$.

Propositions 3.1 and 3.2 establish a relation between problem (38) and the mass optimization problem in both the scalar and vectorial case. Theorem 3.2 shows the relation between the Monge-Kantorovich equations (25) ((27) in the scalar case) and the solutions of the mass optimization problem in both the scalar and the vectorial case (see [23] for the detailed proofs).

The equivalence between (38) and the transportation problem in the scalar case is based on duality again, and it goes back to Kantorovich. We refer to the lectures by Evans [99] and to the work by Evans and Gangbo [100] for the proof of this equivalence, at least in the case $\Omega=\mathbb{R}^{N}, \Sigma=\emptyset, \rho(z)=|z|$, and $f$ satisfying suitable regularity conditions. The general case has been considered by Bouchitté and Buttazzo in [23]. In the vectorial case, even if the equivalence between problem (38), the mass optimization problem, and the 
Monge-Kantorovich equations (25) still holds, it seems that no transportation problem can be identified. In other words, in the vectorial case, the mass optimization problem (15) and the Monge-Kantorovich equations (25) do not seem to be related to the mass transportation with respect to a distance.

For the remaining part of this section we restrict our attention to the scalar case, to a convex open set $\Omega$ and to $j(x)=\frac{1}{2}|z|^{2}$. More general situatios are considered in [23] (see also [64]).

Given an optimal transport plan $\gamma$ it is possible to deduce an optimal density $\mu$ for the mass optimization problem (15) through the formula

$$
\mu(B)=c \int_{\Omega \times \Omega} \mathcal{H}_{x y}^{1}(B) d \gamma(x, y)
$$

where $c$ is a suitable constant and $\mathcal{H}_{x y}^{1}$ denotes the 1-dimensional measure on the segment joining $x$ and $y$.

Vice versa, it is possible to show (see [7]) that given a solution $\mu$ of the mass optimization problem (hence a measure $\lambda$ which solves (19)) there exists an optimal transport plan $\gamma$ such that $\mu$ is associated to $\gamma$ through formula (40) above.

Remark 5.2. A formula like (40) still holds in the case of nonconvex domains $\Omega$, and also in presence of a Dirichlet region $\Sigma$ and with different costs $j$. The difference is that instead of considering the segment joining $x$ to $y$ one has to consider a geodesic $g_{x y}$ for the distance $d_{\Omega, \Sigma}$ introduced in the previous section.

Formula (40) often permits to deduce several properties of the optimal masses and sometimes to identify them directly from the data $f^{+}$and $f^{-}$ (see [23] for some explicit examples). For instance, if we define the geodesic envelope $G\left(f^{+}, f^{-}\right)$of the support of $f$ as the union of all geodesic lines joining a point in the support of $f^{+}$to a point in the support of $f^{-}$we have the following result.

Theorem 5.1. Let $\mu$ be a solution for the mass optimization problem (15). Then the support of $\mu$ is a subset of $G\left(f^{+}, f^{-}\right)$. In particular, if $\Omega$ is convex and $\Sigma=\emptyset$, then

$$
\operatorname{spt} \mu \subset \operatorname{co}\left(\operatorname{spt} f^{+} \cup \operatorname{spt} f^{-}\right),
$$

where co denotes the convex envelope operation.

The following regularity results have been obtained using formula (40) and a property of the support of optimal plans called ciclical monotonicity (see [96], [97]).

Theorem 5.2. Let $\mu$ be a solution for the mass optimization problem 15. Then $\operatorname{dim}(\mu) \geq \max \left\{\operatorname{dim} f^{+}, \operatorname{dim} f^{-}, 1\right\}$. In particular, if $f^{+}\left(\right.$or $\left.f^{-}\right)$is absolutely continuous so is $\mu$. Moreover for every $p \in[1,+\infty]$

$$
f^{+}, f^{-} \in L^{p} \Rightarrow \mu \in L^{p} .
$$


Remark 5.3. The following facts merit to be emphasized:

- It was proved indipendently in [7] and [102] that the optimal density $\mu$ is unique whenever $f^{+}, f^{-} \in L^{1}$. We stress that a similar result is not known in the vectorial case.

- The first summability result for $\mu$ was obtained in [96], where for $1<p<$ $+\infty$ only the $L^{p-\varepsilon}$ regularity was proved. The summability improvement has been recently obtained in [97] by means of elliptic PDE estimates.

- If only one of the two measures $f^{+}$and $f^{-}$is in $L^{p}$ then it can be proved that $\mu \in L^{q}$ for slightly more complicated $q$.

- Nothing is known about the regularity of $\mu$ in the vectorial case.

\section{Further results and open problems}

\subsection{A vectorial example}

Here we present an example of application of the theory developed in the previos sections to a problem of optimal structures in elasticity. We consider the linear isotropic stored energy

$$
j(z)=\frac{\alpha}{2}\left|\operatorname{tr}\left(z^{\text {sym }}\right)\right|^{2}+\beta\left|z^{\text {sym }}\right|^{2}
$$

where $\alpha$ and $\beta$ are the Lamé constants in dimension two.

The problem is the following: distribute in $\mathbb{R}^{2}$ a given amount of mass in order to minimize the elastic compliance related to the force field $f=$ $\delta_{A} \tau_{1}+\delta_{B} \tau_{2}+\delta_{C} \tau_{3}$ which is described in the figure below

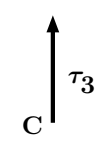

o .
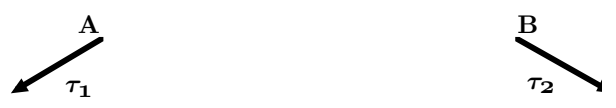

Fig. 3. The force field $f$.

A natural guess is that one of the two structures in the figure below is optimal. 

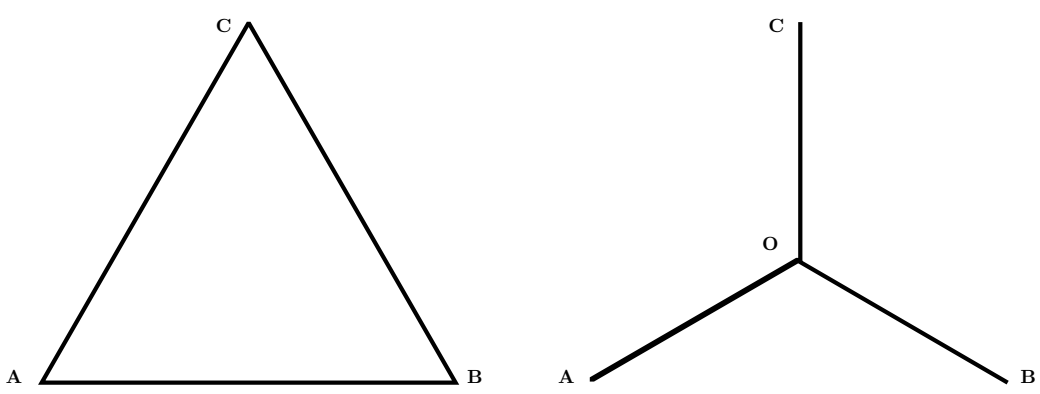

Fig. 4. Two admissible structures.

Indeed it is possible to show that the elastic compliance associated to the two structures is the same and that it is the lowest possible among all 1-dimensional structures. However, none of the two choices is optimal, since a multiple of the optimal mass distribution should satisfy the MongeKantorovich equation and this does not occur for the two structures above (see [23] for a proof). A numerical computation of a two dimensional optimal structure can be found in [109], and the optimal distribution is represented in the figure below.

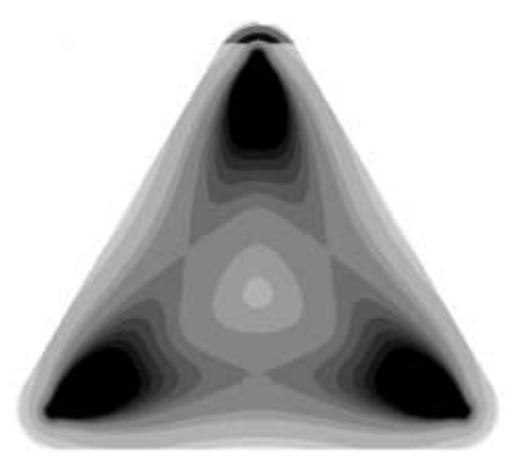

Fig. 5. The optimal mass distribution. 
Remark 6.1. The example above shows that Theorem 5.1 does not hold in the vectorial case. A weaker formulation, which could be expected to hold in the vectorial case, is:

$$
\text { spt } f \text { compact } \Rightarrow \operatorname{spt} \mu \text { compact. }
$$

We do not know to prove (or disprove) this property.

\subsection{A $p$-Laplacian approximation}

We now discuss some approximation of the mass optimization problem which is naturaly suggested by Remark 3.3. Indeed, as the optimal mass does not depend on the growth exponent of $j$, which we recall can be written as $j(z)=$ $\frac{1}{p} \rho(z)^{p}$, it is natural to study the asymptotics for $p \rightarrow \infty$ of

$$
F_{p}(u)= \begin{cases}\frac{1}{p} \int_{\Omega} \rho(e(u))^{p} d x-\langle f, u\rangle & \text { if } u \in W^{1, p}\left(\Omega, \mathbb{R}^{N}\right) \text { and } u=0 \text { on } \Sigma \\ +\infty & \text { otherwise. }\end{cases}
$$

What we espect is that problem

$$
\min _{u} F_{p}(u)
$$

converges, when $p \rightarrow+\infty$, to

$$
\min \{-\langle f, u\rangle: \rho(e(u)) \leq 1 \text { a.e., } u=0 \text { on } \Sigma\},
$$

whose relationships with the mass optimization problem have been explained in the previous sections of these notes. As the dual problem of (44) is also involved in the mass optimization problem, it is natural to look also at the asymptotic behaviour of the dual problem of (43) which is:

$$
\min \left\{\frac{1}{p^{\prime}} \int_{\Omega}\left(\rho^{0}(\sigma)\right)^{p^{\prime}} d x: \sigma \in L^{p^{\prime}}\left(\Omega, \mathbb{R}^{N \times N}\right),-\operatorname{div} \sigma=f \text { in } \Omega \backslash \Sigma\right\} .
$$

Notice that the duality relationships says that if $u_{p}$ is a minimizer of $F_{p}$ then any minimizer of (45) can be written in the form $\sigma_{p}=\xi_{p} \rho\left(e\left(u_{p}\right)\right)^{p-2}$ where $\xi_{p}$ is an element of $\partial\left(\rho\left(e\left(u_{p}\right)\right)\right)$. The convergence result proved in [24] is:

Theorem 6.1. Assume that the minimum in (44) is finite and let $\left\{\left(u_{p}, \sigma_{p}\right)\right\}$ be a sequence of minimizers for (43) and (45). Set:

$$
\mu_{p}:=\rho\left(e\left(u_{p}\right)\right)^{p-2}, \quad \sigma_{p}=\xi_{p} \mu_{p}
$$

Then there exist $u \in \operatorname{Lip}_{1, \rho}(\Omega, \Sigma), \mu \in \mathcal{M}(\bar{\Omega})$ and a suitable rigid displacement $r_{p} \in \mathcal{R}_{\Sigma}$, such that, up to subsequences:

(i) $u_{p}-r_{p} \rightarrow u$ uniformly; 
(ii) $\mu_{p} \rightarrow \mu$ in $\mathcal{M}(\bar{\Omega})$;

(iii) $\xi_{p} \mu_{p} \rightarrow \xi \mu$ in $\mathcal{M}\left(\bar{\Omega}, \mathbb{R}^{N}\right)$;

(iv) $u$ is a minimizer of (44) and $(u, \xi, \mu)$ is a solution of the MongeKantorovich equation.

Moreover, if the set $\left\{\rho^{0} \leq 1\right\}$ is strictly convex, then the convergence $\xi_{p} \rightarrow \xi$ holds in a stronger sense.

Remark 6.2. In the scalar case of conductivity, Theorem 6.1 can be reformulated as follows: assume that $\Sigma \neq \emptyset$ (or that $\int d f=0$ ) and let $u_{p}$ be the unique solution (up to an additive constant) of:

$$
-\Delta_{p} u_{p}=f \quad \text { on } \Omega \backslash \Sigma, \quad u_{p}=0 \quad \text { on } \Sigma, \quad \frac{\partial u_{p}}{\partial \nu}=0 \quad \text { on } \partial \Omega \backslash \Sigma .
$$

Then up to subsequences, we have:

$$
\begin{aligned}
& u_{p} \rightarrow u \text { uniformly, } \\
& \left|\nabla u_{p}\right|^{p-2} \rightarrow \mu \text { in } \mathcal{M}(\bar{\Omega}), \\
& \left|\nabla u_{p}\right|^{p-2} \nabla u_{p} \rightarrow \mu \nabla_{\mu} u \quad \text { in } \mathcal{M}\left(\bar{\Omega}, \mathbb{R}^{N}\right),
\end{aligned}
$$

where $(u, \mu)$ solves the scalar Monge-Kantorovich equation.

Remark 6.3. In the scalar case and for $f$ regular enough the approximation above has have been used by Evans and Gangbo in [100] to prove one of the first existence results for the Monge problem.

Remark 6.4. The main difference between the scalar and the vectorial case is that in the vectorial case one has to assume that the limit problem admits a minimizer to obtain the approximation result (6.1) while in the scalar case this is a consequence of the approximation. This is due to the fact that the Korn's inequality (needed in the vectorial case) does not hold in $W^{1, \infty}$ and then it is not stable in $W^{1, p}$ when $p \rightarrow \infty$. In the scalar case the main role is played by the Poincaré inequality which is more stable.

\subsection{Optimization of Dirichlet regions}

If we consider the cost in (33), (35)

$$
J(\Sigma)=\min \left\{\int_{\bar{\Omega} \times \bar{\Omega}} \psi\left(d_{\Omega, \Sigma}(x, y)\right) d \gamma(x, y): \gamma \in P\left(f^{+}, f^{-}\right)\right\}
$$

as a function of $\Sigma$ only, once $f^{+}, f^{-}, \Omega, \psi$ are fixed, a natural question which arises is to optimize the functional $J$ in a suitable class of admissible Dirichlet regions $\Sigma$. To this kind of problems belong several questions, known in the literature under different names. We list here below some of them.

Location problems. In this setting $f^{-} \equiv 0$ and $f^{+} \in L^{1}(\Omega)$, so that the functional $J(\Sigma)$ in (46) simply becomes 


$$
J(\Sigma)=\int_{\Omega} \psi\left(\operatorname{dist}_{\Omega}(x, \Sigma)\right) f^{+}(x) d x .
$$

The admissible $\Sigma$ are the subsets of $\bar{\Omega}$ made of $n$ points; hence the optimal location problem reads as

$$
\min \left\{\int_{\Omega} \psi\left(\operatorname{dist}_{\Omega}(x, \Sigma)\right) f^{+}(x) d x: \Sigma \subset \bar{\Omega}, \# \Sigma=n\right\}
$$

where $\operatorname{dist}_{\Omega}(x, \Sigma)$ is the distance of $x$ to $\Sigma$ measured in the geodesic metric of $\Omega$. Problem (48) describes the question of locating in a given region $\Omega$ a given number $n$ of points of distribution of a certain product, in order to minimize the average distance (in the case $\psi(d)=d$ ) that customers, whose density $f^{+}(x)$ in $\Omega$ is known, have to cover to reach the closest point of distribution.

The existence of an optimal $\Sigma_{n}$, for a fixed $n$, is straightforward. It is also clear that the minimum value $I_{n}$ of problem (48) tends to zero as $n \rightarrow+\infty$, and a simple argument allows us to obtain that

$$
I_{n}=O\left(\psi\left(n^{-1 / N}\right)\right) .
$$

Hence it is interesting to study the asymptotic behaviour (as $n \rightarrow+\infty$ ) of the rescaled functionals

$$
J_{n}(\Sigma)= \begin{cases}J(\Sigma) / I_{n} & \text { if } \# \Sigma=n \\ +\infty & \text { otherwise }\end{cases}
$$

in terms of the $\Gamma$-convergence, with respect to the convergence defined on the space of measures by

$$
\Sigma_{n} \rightarrow \lambda \quad \Longleftrightarrow \quad \frac{1}{n} \mathcal{H}^{0}\left\llcorner\Sigma_{n} \rightarrow \lambda \quad\right. \text { weakly* }
$$

A reasonable conjecture, in the case $\Omega$ convex and $\psi(d)=d$, also supported by the results of [132], is that the $\Gamma$-limit functional is written as

$$
J_{\infty}(\lambda)=C \int f^{+}(x) \lambda^{-1 / N}
$$

intended in the sense of convex functionals over the measures, where $C$ is a suitable constant. In particular, this would imply that the best location of $n$ points in $\Omega$ has to be asymptotically proportional to $\left(f^{+}(x)\right)^{N /(N+1)}$, being $f^{+}(x)$ the density of population in $\Omega$.

It would also be interesting to compute how good, with respect to the optimal choice $\Sigma_{n}$, is a random choice of $n$ points in $\Omega$.

Irrigation problems. Take again $f^{-} \equiv 0$ and $f^{+} \in L^{1}(\Omega)$, which gives $J(\Sigma)$ as in (47). The admissible $\Sigma$ are now the closed connected subsets of $\bar{\Omega}$ whose 1-dimensional Hausdorff measure $\mathcal{H}^{1}$ does not exceed a given number $L$. The optimization problem then reads as 


$$
\min \left\{J(\Sigma): \Sigma \subset \bar{\Omega}, \Sigma \text { closed connected, } \mathcal{H}^{1}(\Sigma) \leq L\right\}
$$

In other words we want to irrigate the region $\Omega$ by a system of water channels $\Sigma$ and the cost of irrigating a point $x \in \Omega$, where a mass $f^{+}(x) d x$ is present, is assumed to be proportional to $\psi\left(\operatorname{dist}_{\Omega}(x, \Sigma)\right)$.

The existence of an optimal $\Sigma_{L}$ easily follows from the Golab theorem (see [64]) and several qualitative properties of $\Sigma_{L}$ can be shown. We refer to [64] for a more complete analysis and open problems about the structure of $\Sigma_{L}$. Here we want simply to stress that an asymptotic study (as $L \rightarrow+\infty$ ), similar to the one presented above for location problems, seems interesting; however, if $I_{L}$ denotes the minimum value of problem (49), it is still possible to obtain, at least when $\psi(d)=d$, the asymptotic estimate (see [64])

$$
I_{L}=O\left(L^{1 /(1-N)}\right) \text {. }
$$

This makes reasonable the conjecture that the $\Gamma$-limit functional (as $L \rightarrow$ $+\infty)$, with respect to a convergence on $\Sigma_{L}$ similar to the one above, takes the form

$$
J_{\infty}(\lambda)=K \int f^{+}(x) \lambda^{1 /(1-N)}
$$

for a suitable constant $K$. Again, this would imply that the density of the optimal irrigation channels is asymptotically (as $L \rightarrow+\infty$ ) proportional to $\left(f^{+}(x)\right)^{(N-1) / N}$.

Problems in urban planning. Here $f^{+}$represents the density of the working population in an urban area $\Omega$, and $f^{-}$the density of the working places, that are assumed to be known. Notice that in several problems in urban planning $f^{+}$and $f^{-}$are the main unknowns and the optimal location of them in a given region $\Omega$ is obtained through the minimization of a cost functional which takes into account a penalization for concentrated $f^{+}$, a penalization for sparse $f^{-}$, and the cost of transporting $f^{+}$onto $f^{-}$.

The closed connected subset $\Sigma$ of $\bar{\Omega}$ represents the urban transportation network that has to be designed in order to minimize the cost $J(\Sigma)$ in (46). The problem then becomes

$$
\min \left\{J(\Sigma): \Sigma \subset \bar{\Omega}, \Sigma \text { closed connected, } \mathcal{H}^{1}(\Sigma) \leq L\right\}
$$

In this model the population is transported on $\Sigma$ for free, but it is possible to consider similar models where customers pay a ticket which depends in some way on the length of the part of $\Sigma$ they used.

\subsection{Optimal transporting distances}

A different kind of optimization problem related to mass transportation consists in considering the marginal measures $f^{+}$and $f^{-}$as given and to searching 
a distance $d$ which optimizes the transportation cost among all distances belonging to some admissible class. More precisely, we consider a domain $\Omega$ of $\mathbb{R}^{N}$, that for simplicity we take convex, and for every Borel coefficient $a(x)$ the corresponding Riemannian distance

$$
d_{a}(x, y)=\inf \left\{\int_{0}^{1} a(\gamma(t))\left|\gamma^{\prime}(t)\right| d t: \gamma(0)=x, \gamma(1)=y\right\} .
$$

A small coefficient $a(x)$ in a region $\omega$ makes the transportation in $\omega$ easy, while on the contrary, large coefficients make the transportation more costly.

The admissible class we shall take into consideration is the class of distances of the form $d_{a}$ where the coefficient $a$ varies into

$$
\mathcal{A}=\left\{\alpha \leq a(x) \leq \beta, \int_{\Omega} a(x) d x \leq m\right\}
$$

being $\alpha, \beta, m$ given constants, satisfying the compatibility condition

$$
\alpha|\Omega| \leq m \leq \beta|\Omega|
$$

For every $a \in \mathcal{A}$ we consider the mass transportation cost

$$
F(a)=\inf \left\{\int_{\bar{\Omega} \times \bar{\Omega}} \psi\left(d_{a}(x, y)\right) d \gamma(x, y): \pi_{\sharp}^{1} \gamma=f^{+}, \pi_{\sharp}^{2} \gamma=f^{-}\right\}
$$

where $\psi:[0,+\infty[\rightarrow[0,+\infty[$ is a continuous and nondecreasing function.

The minimization problem for $F$ on $\mathcal{A}$, which correspond to find the most favourable coefficient for the transportation of $f^{+}$onto $f^{-}$, is somehow trivial. In fact, it is easy to show that

$$
\inf \{F(a): a \in \mathcal{A}\}=F(\alpha) \text {. }
$$

On the contrary, the problem of maximizing the functional $F$ on $\mathcal{A}$ seems interesting. In other words, we want to prevent as much as possible the transportation of $f^{+}$onto $f^{-}$, making its cost as big as possible, playing on the Riemannian coefficient $a(x)$. Notice that the integral constraint $\int_{\Omega} a(x) d x \leq m$ forces us to use carefully the quantity $m$ (a kind of total prevention power) at our disposal: we do not have to waste high coefficients in regions which are not essential for the mass transportation

The first attempt to find a solution of the maximization problem

$$
\max \{F(a): a \in \mathcal{A}\}
$$

consists in trying to use the direct methods of the calculus of variations: this requires to introduce a topology on the class of admissible choices strong enough to have the lower semicontinuity of the cost functional and weak enough to have the compactness of minimizing sequences. In our case the best choice would be to relate the convergence of a sequence of coefficients $\left(a_{n}\right)$ to the 
convergence of geodesic paths corresponding to the distances $d_{a_{n}}$; more precisely, since the geodesic paths could be nonunique, the definition has to be given in terms of $\Gamma$-convergence for the length functionals $L_{n}$ with respect to $d_{a_{n}}$. Here we mean that for every distance $d$ we may consider the length functional $L_{d}$ defined on curves $\gamma:[0,1] \rightarrow \Omega$ by

$$
L_{d}(\gamma)=\sup \left\{\sum_{i=1}^{k} d\left(\gamma\left(t_{i}\right), \gamma\left(t_{i+1}\right): t_{1}<\cdots<t_{k}\right\} .\right.
$$

Thus we define the convergence $\tau$ on the class of geodesic distances (that is the ones whose value between two points coincides with the infimum of the lengths of all paths joining the two points) by setting

$$
d_{n} \rightarrow d \text { in } \tau \quad \Longleftrightarrow \quad L_{d_{n}} \rightarrow L_{d} \text { in the } \Gamma \text {-convergence }
$$

where we considered the family of curves $\gamma$ endowed with the uniform convergence.

It is not difficult to show that with this choice of convergence the functional $F(a)$ turns out to be continuous on $\mathcal{A}$; moreover (see [58]) we have that a sequence $d_{n}$ converges in $\tau$ to $d$ if and only if $d_{n}$ converges to $d$ uniformly as a sequence of functions in $\Omega \times \Omega$ (see also [65], where the uniform convergence $d_{n} \rightarrow d$ is related to the $\Gamma$-convergence of the onedimensional Hausdorff measures $\mathcal{H}_{d_{n}}^{1} \rightarrow \mathcal{H}_{d}^{1}$ ). Therefore, due to the upper bound $\beta$, all distances $d_{a}$ with $a \in \mathcal{A}$ are equi-Lipschitz continuous, so that Ascoli-Arzelà theorem allows to conclude that the class $\mathcal{A}$ is pre-compact for the $\tau$ convergence.

Unfortunately, we cannot conclude by the usual argument of the direct methods of the calculus of variations because the class $\mathcal{A}$ is not closed under $\tau$. This is known since a long time (see [1]) through the simple example of a chessboard structure in $\mathbb{R}^{2}$ of side $\varepsilon$, with $a_{\varepsilon}(x)=\alpha$ on white cells and $a_{\varepsilon}(x)=\beta$ on black cells, whose associated distances $d_{a_{\varepsilon}}$ converge, in the sense above, to a distance $d$ which is not of Riemannian type. The explicit computation of this limit $d$ can be easily made when the ratio $\beta / \alpha$ is large enough, and in this case we find that $d$ is a Finsler distance generated by a Finsler metric $\phi(z)$ (a convex and 1-homogeneous function) whose unit ball is a regular octagone.

On the other hand, it is known (see [153]) that the class of geodesic distances coincides with the class of Finsler distances, through the derivation formula

$$
\phi(x, z)=\limsup _{t \rightarrow 0} \frac{d(x+t z, x)}{t} .
$$

More recently, in [29] it has been actually proved a density result for the distances of the form $d_{a}$, with $\alpha \leq a \leq \beta$, in the class of all Finsler distances $d$ generated by metrics $\phi(x, z)$, which leads us to expect that the relaxed maximization problem associated to (52) is defined on the $\tau$ closure $\overline{\mathcal{A}}$ of $\mathcal{A}$, which is a subclass of Finsler distances on $\Omega$ : 


$$
\max \{\mathcal{F}(d): d \in \overline{\mathcal{A}}\}
$$

being $\mathcal{F}(d)$ defined as in $(51)$ with $d$ which replaces $d_{a}$. However, we are able to prove that the original maximization problem (52) actually admits an optimal unrelaxed solution, thanks to the following lemma (see [57]).

Lemma 6.1. Let $\phi(x, z)$ be a Finsler metric which comes out as a limit of a sequence $\left(a_{n}\right)$ of coefficients in the original admissible class $\mathcal{A}$. Then, if we define the largest eigenvalue $\Lambda_{\phi}(x)$ of $\phi(x, \cdot)$ as

$$
\Lambda_{\phi}(x)=\sup \{\phi(x, z):|z| \leq 1\}
$$

we have that

$$
\int_{\Omega} \Lambda_{\phi}(x) d x \leq m .
$$

The proof of the existence of an optimal unrelaxed solution for the original maximization problem (52) follows now easily.

Theorem 6.2. The original maximization problem (52) admits an optimal solution.

Proof. Take a maximizing sequence $\left(a_{n}\right)$; according to what seen above we may find a subsequence (still denoted by the same indices) which $\tau$ converges to a Finsler distance $d$ generated by a Finsler metric $\phi(x, z)$. We have then

$$
\mathcal{F}(d)=\lim _{n \rightarrow+\infty} F\left(a_{n}\right)
$$

By the definition of the largest eigenvalue $\Lambda_{\phi}(x)$ we have

$$
\phi(x, z) \leq \Lambda_{\phi}(x)|z|
$$

Using now the fact that the cost functional is monotone increasing with respect to the distance functions, we may conclude that the coefficient $a(x)=$ $\Lambda_{\phi}(x)|z|$, which is admissible for our problem thanks to Lemma 6.1, maximizes the functional $F$ on the class $\mathcal{A}$.

Acknowledgements. These notes have been written as a development of a course given by G. B. at the CIME Summer School "Optimal Transportation and Applications" held in Martina Franca on September 2001. We wish to thank the CIME for the excellent organization and for the friendly and constructive atmosphere during the School. This work is also part of the European Research Training Network "Homogenization and Multiple Scales" (HMS2000) under contract HPRN-2000-00109. 


\section{References}

1. E. ACERBI, G. BUTTAZZO: On the limits of periodic Riemannian metrics. J. Analyse Math., 43 (1984), 183-201.

2. E. ACERBI, G. BUTTAZZO: Reinforcement problems in the calculus of variations. Ann. Inst. H. Poincaré Anal. Non Linéaire, 3 (4) (1986), 273-284.

3. G. ALLAIRE: Shape optimization by the homogeneization method. Applied Mathematical Sciences 146, Springer-Verlag, New York (2002).

4. G. ALLAIRE, E. BONNETIER, G. FRANCFORT, F.JOUVE: Shape optimization by the homogenization method. Numer. Math. 76 (1997), 27-68.

5. G. ALLAIRE, R. V. KOHN: Optimal design for minimum weight and compliance in plane stress using extremal microstructures. Europ. J. Mech. A/Solids, 12 (6) (1993), 839-878.

6. L. AMBROSIO: Introduction to geometric measure theory and minimal surfaces (Italian), Scuola Normale Superiore, Pisa (1997).

7. L. AMBROSIO: Lecture notes on optimal transport problems. Preprint n. 32, Scuola Normale Superiore, Pisa (2000).

8. L. AMBROSIO, G. BUTTAZZO: An optimal design problem with perimeter penalization. Calc. Var., 1 (1993), 55-69.

9. L. AMBROSIO, N. FUSCO, D. PALLARA: Functions of bounded variation and free discontinuity problems. Oxford Mathematical Monographs, Clarendon Press, Oxford (2000).

10. L. AMBROSIO, A. PRATELLI: Existence and stability results in the $L^{1}$ theory of optimal transportation. Preprint Scuola Normale Superiore, Pisa (2002).

11. L. AMBROSIO, P. TILLI: Selected Topics on Analysis in Metric Spaces. Scuola Normale Superiore, Pisa, (2000).

12. M. S. ASHBAUGH: Open problems on eigenvalues of the Laplacian. Analytic and geometric inequalities and applications, 13-28, Math. Appl., 478, Kluwer Acad. Publ., Dordrecht (1999).

13. H. ATTOUCH: Variational Convergence for Functions and Operators. Pitman, Boston (1984).

14. H. ATTOUCH, G. BUTTAZZO: Homogenization of reinforced periodic onecodimensional structures. Ann. Scuola Norm. Sup. Pisa Cl. Sci., 14 (1987), $465-484$.

15. D. AZE, G. BUTTAZZO: Some remarks on the optimal design of periodically reinforced structures. RAIRO Modél. Math. Anal. Numér., 23 (1989), 53-61.

16. E. BAROZZI, E. H. A. GONZALEZ: Least area problems with a volume constraint. In "Variational methods for equilibrium problems of fluids", Astérisque 118, (1984), 33-53.

17. M. BELliEUD, G. BOUCHITTE: Homogenization of elliptic problems in a fiber reinforced structure. Nonlocal effects. Ann. Scuola Norm. Sup. Pisa Cl. Sci., 26 (1998), 407-436.

18. M. BELLONI, G. BUTTAZZO, L. FREDDI: Completion by Gammaconvergence for optimal control problems. Ann. Fac. Sci. Toulouse Math., 2 (1993), 149-162.

19. M. BELLONI, B. KAWOHL: A paper of Legendre revisited. Forum Math., 9 (1997), 655-667.

20. M. BELLONI, A. WAGNER: Newton's problem of minimal resistance in the class of bodies with prescribed volume. Preprint Università di Parma, Parma (2001). 
21. J. D. BENAMOU, Y. BRENIER: Mixed $L^{2}$-Wasserstein optimal mapping between prescribed density functions. J. Optim. Theory Appl., 111 (2001), 255271.

22. M. BENDSØE: Optimal shape design as a material distribution problem. Struct. Optim., 1 (1989), 193-202.

23. G. BOUCHITTE, G. BUTTAZZO: Characterization of optimal shapes and masses through Monge-Kantorovich equation. J. Eur. Math. Soc., 3 (2001), $139-168$.

24. G. BOUChitTE, G. BUTTAZZO, L. DE PASCALE: A p-Laplacian approximation for some mass optimization problems. J. Optim. Theory Appl., (to appear).

25. G. BOUChitte, G. BUtTAZZO, I. FRAGALÀ: Convergence of Sobolev spaces on varying manifolds. J. Geom. Anal., 11 (2001), 399-422.

26. G. BOUCHITTE, G. BUTTAZZO, I. FRAGALÀ: Bounds for the effective coefficients of homogenized low dimensional structures. J. Math. Pures Appl, (to appear).

27. G. BOUCHITTE, G. BUTTAZZO, P. SEPPECHER: Energies with respect to a measure and applications to low dimensional structures. Calc. Var., 5 (1997), $37-54$.

28. G. BOUCHITTE, G. BUTTAZZO, P. SEPPECHER: Shape optimization solutions via Monge-Kantorovich equation. C. R. Acad. Sci. Paris, 324-I (1997), $1185-1191$.

29. A. BRAIDES, G. BUTTAZZO, I. FRAGALÀ: Riemannian approximation of Finsler metrics. Asymptotic Anal. (to appear).

30. H. BREZIS: Liquid crystals and energy estimates for $S^{2}$-valued maps. In "Theory and applications of liquid crystals", IMA Vol. Math. Appl. 5, SpringerVerlag, New York (1987), 31-52.

31. H. BREZIS: $S^{k}$-valued maps with singularities. In "Topics in calculus of variations", Lecture Notes in Math. 1365, Springer-Verlag, Berlin (1989), 1-30.

32. H. BREZIS, J. M. CORON, E. H. LIEB: Harmonic maps with defects. Commun. Math. Phys., 107 (1986), 649-705.

33. T. BRIANÇON: Problèmes de régularité en optimisation de formes. Ph.D thesis, ENS Cachan-Bretagne (2002).

34. F. BROCK, V. FERONE, B. KAWOHL: A symmetry problem in the calculus of variations. Calc. Var. Partial Differential Equations, 4 (1996), 593-599.

35. D. BUCUR: Shape analysis for Nonsmooth Elliptic Operators. Appl. Math. Lett., 9 (1996), 11-16.

36. D. BUCUR, G. BUTTAZZO: Results and questions on minimum problems for eigenvalues. Preprint Dipartimento di Matematica Università di Pisa, Pisa (1998).

37. D. BUCUR, G. BUTTAZZO: Variational Methods in some Shape Optimization Problems. Lecture Notes of courses at Dipartimento di Matematica Università di Pisa and Scuola Normale Superiore di Pisa, Series "Appunti della Scuola Normale Superiore", Pisa (2002).

38. D. BUCUR, G. BUTTAZZO, I. FIGUEIREDO: On the attainable eigenvalues of the Laplace operator. SIAM J. Math. Anal., 30 (1999), 527-536.

39. D. BUCUR, G. BUTTAZZO, A. HENROT: Existence results for some optimal partition problems. Adv. Math. Sci. Appl., 8 (1998), 571-579.

40. D. BUCUR, G. BUTTAZZO, P. TREBESCHI: An existence result for optimal obstacles. J. Funct. Anal., 162 (1999), 96-119. 
41. D. BUCUR, G. BUTTAZZO, N. VARCHON: On the problem of optimal cutting, SIAM J. Optimization (to appear).

42. D. BUCUR, A. HENROT: Minimization of the third eigenvlaue of the Dirichlet Laplacian. Proc. Roy. Soc. London, Ser. A, 456 (2000), 985-996.

43. D. BUCUR, A. HENROT: Stability for the Dirichlet problem under continuous Steiner symmetrization. Potential Anal., 13 (2000), 127-145.

44. D. BUCUR, A. HENROT, J. SOKOLOWSKI, A. ZOCHOWSKI: Continuity of the elasticity system solutions with respect to boundary variations. Adv. Math. Sci. Appl., 11 (2001), 57-73.

45. D. BUCUR, P. TREBESCHI: Shape optimization problem governed by nonlinear state equation. Proc. Roy. Soc. Edinburgh, 128 A (1998), 945-963.

46. D. BUCUR, N. VARCHON: Boundary variation for the Neumann problem, Ann. Scuola Norm. Sup. Pisa Cl. Sci., XXIV (2000), 807-821.

47. D. BUCUR, N. VARCHON: A duality approach for the boundary variations of Neumann problems, Preprint Université de Franche-Comté n. 00/14, Besançon (2000).

48. D. BUCUR, J. P. ZOLESIO: N-Dimensional Shape Optimization under Capacitary Constraints. J. Differential Equations, 123 (2) (1995), 504-522.

49. D. BUCUR, J. P. ZOLESIO: Shape continuity for Dirichlet-Neumann problems. Progress in partial differential equations: the Metz surveys, 4, Pitman Res. Notes Math. Ser. 345, Longman, Harlow (1996), 53-65.

50. D. BUCUR, J. P. ZOLESIO Wiener's criterion and shape continuity for the Dirichlet problem. Boll. Un. Mat. Ital. B (7) 11 (1997), 757-771.

51. G. BUTTAZZO: Thin insulating layers: the optimization point of view. Proceedings of "Material Instabilities in Continuum Mechanics and Related Mathematical Problems", Edinburgh 1985-1986, edited by J. M. Ball, Oxford University Press, Oxford (1988), 11-19.

52. G. BUTTAZZO: Semicontinuity, Relaxation and Integral Representation in the Calculus of Variations. Pitman Res. Notes Math. Ser. 207, Longman, Harlow (1989).

53. G. BUTTAZZO, G. DAL MASO: Shape optimization for Dirichlet problems: relaxed solutions and optimality conditions. Bull. Amer. Math. Soc., 23 (1990), 531-535.

54. G. BUTTAZZO, G. DAL MASO: Shape optimization for Dirichlet problems: relaxed formulation and optimality conditions. Appl. Math. Optim., 23 (1991), $17-49$.

55. G. BUTTAZZO, G. DAL MASO: An existence result for a class of shape optimization problems. Arch. Rational Mech. Anal., 122 (1993), 183-195.

56. G. BUTTAZZO, G. DAL MASO, A. GARRONI, A. MALUSA: On the relaxed formulation of Some Shape Optimization Problems. Adv. Math. Sci. Appl., 7 (1997), 1-24.

57. G. BUTTAZZO, A. DAVINI, I. FRAGALÀ, F. MACIÁ: Optimal Riemannian distances preventing mass transfer. Preprint Dipartimento di Matematica Università di Pisa, Pisa (2002).

58. G. BUTTAZZO, L. DE PASCALE, I. FRAGALÀ: Topological equivalence of some variational problems involving distances. Discrete and Continuous Dynamical Systems, 7 (2001), 247-258.

59. G. BUTTAZZO, V. FERONE, B. KAWOHL: Minimum problems over sets of concave functions and related questions. Math. Nachr., 173 (1995), 71-89. 
60. G. BUTTAZZO, L. FREDDI: Relaxed optimal control problems and applications to shape optimization. Lecture notes of a course held at the NATO-ASI Summer School "Nonlinear Analysis, Differential Equations and Control", Montreal, July 27 - August 7, 1998, Kluwer, Dordrecht (1999), 159-206.

61. G. BUTTAZZO, M. GIAQUINTA, S. HILDEBRANDT: One-dimensional Calculus of Variations: an Introduction. Oxford University Press, Oxford (1998).

62. G. BUTTAZZO, P. GUASONI: Shape optimization problems over classes of convex domains. J. Convex Anal., 4 (1997), 343-351.

63. G. BUTTAZZO, B. KAWOHL: On Newton's problem of minimal resistance. Math. Intelligencer, 15 (1993), 7-12.

64. G. BUTTAZZO, E. OUDET, E. STEPANOV: Optimal transportation problems with free Dirichlet regions. Preprint Dipartimento di Matematica Università di Pisa, Pisa (2002).

65. G. BUTTAZZO, B. SCHWEIZER: $\Gamma$ convergence of Hausdorff measures. Preprint Dipartimento di Matematica Università di Pisa, Pisa (2002).

66. G. BUTTAZZO, P. TREBESCHI: The role of monotonicity in some shape optimization problems. In "Calculus of Variations, Differential Equations and Optimal Control", Research Notes in Mathematics Series, Vol. 410-411, Chapman \& Hall/CRC Press, Boca Raton (1999).

67. G. BUTTAZZO, A. WAGNER: On the optimal shape of a rigid body supported by an elastic membrane. Nonlinear Anal., 39 (2000), 47-63.

68. G. BUTTAZZO, O. M. ZEINE: Un problème d'optimisation de plaques. Modél. Math. Anal. Numér., 31 (1997), 167-184.

69. E. CABIB: A relaxed control problem for two-phase conductors. Ann. Univ. Ferrara - Sez. VII - Sc. Mat., 33 (1987), 207-218.

70. E. CABIB, G. DAL MASO: On a class of optimum problems in structural design. J. Optimization Theory Appl., 56 (1988), 39-65.

71. G. CARLIER, T. LACHAND-ROBERT: Regularity of solutions for some variational problems subject to a convexity constraint. Comm. Pure Appl. Math., 54 (2001), 583-594.

72. J. CEA, K. MALANOWSKI: An example of a max-min problem in partial differential equations. SIAM J. Control, 8 (1970), p. 305-316.

73. A. CHAMBOLLE, F. DOVERI: Continuity of Neumann linear elliptic problems on varying two-dimensional bounded open sets., Commun. Partial Differ. Equations, 22 (1997), 811-840.

74. A. CHAMBOLLE: $A$ density result in two-dimensional linearized elasticity and applications, Preprint Ceremade, Paris (2001).

75. D. CHENAIS: On the existence of a solution in a domain identification problem. J. Math. Anal. Appl., 52 (1975), 189-219.

76. D. CHENAIS: Homéomorphisme entre ouverts lipschitziens. Ann. Mat. Pura Appl. (4) 118 (1978), 343-398.

77. M. CHIPOT, G. DAL MASO: Relaxed shape optimization: the case of nonnegative data for the Dirichlet problem. Adv. Math. Sci. Appl., 1 (1992), 47-81.

78. D. CIORANESCU, F. MURAT: Un terme étrange venu d'ailleurs. Nonlinear partial differential equations and their applications. Collège de France Seminar, Vol. II (Paris, 1979/1980), pp. 98-138, 389-390, Res. Notes in Math., 60, Pitman, Boston, Mass.-London (1982).

79. M. COMTE, T. LACHAND-ROBERT: Existence of minimizers for Newton's problem of the body of minimal resistance under a single impact assumption. J. Anal. Math. 83 (2001), 313-335. 
80. M. COMTE, T. LACHAND-ROBERT: Newton's problem of the body of minimal resistance under a single-impact assumption. Calc. Var. Partial Differential Equations 12 (2001), 173-211.

81. S. J. COX: The shape of the ideal column. Math. Intelligencer, 14 (1992), 16-24.

82. B. DACOROGNA: Direct Methods in the Calculus of Variations. Appl. Math. Sciences 78, Springer-Verlag, Berlin (1989).

83. G. DAL MASO: An Introduction to $\Gamma$-convergence. Birkhäuser, Boston (1993).

84. G. DAL MASO, A. DE FRANCESCHI: Limits of nonlinear Dirichlet problems in varying domains. Manuscripta Math., 61 (1988), 251-268.

85. G. DAL MASO, A. GARRONI: New results on the asymptotic behaviour of Dirichlet problems in perforated domains. Math. Mod. Meth. Appl. Sci., 3 (1994), 373-407.

86. G. DAL MASO, A. MALUSA: Approximation of relaxed Dirichlet problems by boundary value problems in perforated domains. Proc. Roy. Soc. Edinburgh Sect. A-125 (1995), 99-114.

87. G. DAL MASO, F. MURAT: Asymptotic behavior and correctors for Dirichlet problems in perforated domains with homogeneous monotone operators. Ann. Scuola Norm. Sup. Pisa, 24 (1997), 239-290.

88. G. DAL MASO, R. TOADER: A model for the quasi-static growth of a brittle fracture: existence and approximation results. Preprint SISSA, Trieste (2001).

89. E. DE GIORGI: Teoremi di semicontinuità nel calcolo delle variazioni. Notes of a course given at the Istituto Nazionale di Alta Matematica, Rome (1968).

90. E. DE GIORGI: $\Gamma$-convergenza e $G$-convergenza. Boll. Un. Mat. Ital., 14-A (1977), 213-224.

91. E. DE GIORGI, F. COLOMBINI, L.C. PICCININI: Frontiere orientate di misura minima e questioni collegate. Quaderni della Scuola Normale Superiore, Pisa (1972).

92. E. DE GIORGI, T. FRANZONI: Su un tipo di convergenza variazionale. Atti Accad. Naz. Lincei Cl. Sci. Fis. Mat. Natur., (8) 58 (1975), 842-850.

93. M. DELFOUR, J.-P. ZOLESIO: Shapes and geometries. Analysis, differential calculus, and optimization. Advances in Design and Control (SIAM), Philadelphia (2001).

94. F. DEMENGEL: Déplacements à déformations bornées et champs de contrainte mesures. Ann. Scuola Norm. Sup. Pisa Cl. Sci., 12 (1985), 243-318.

95. F. DEMENGEL, P. SUQUET: On locking materials. Acta Applicandae Math., 6 (1986), 185-211.

96. L. DE PASCALE, A. PRATELLI: Regularity properties for Monge transport density and for solutions of some shape optimization problem. Calc. Var., 14 (2002), 249-274.

97. L. DE PASCALE, L. C. EVANS, A. PRATELLI: Integral estimates for transport densities. Work in preparation.

98. I. EKELAND, R. TEMAM: Convex Analysis and Variational Problems. Studies in Mathematics and its Applications 1, North-Holland, Amsterdam (1976).

99. L. C. EVANS: Partial differential equations and Monge-Kantorovich mass transfer. Current Developments in Mathematics, Cambridge MA (1997), 65-126, Int. Press, Boston (1999).

100. L. C. EVANS, W. GANGBO: Differential Equations Methods for the MongeKantorovich Mass Transfer Problem. Mem. Amer. Math. Soc. 137, Providence (1999). 
101. H. FEDERER: Geometric Measure Theory. Springer-Verlag, Berlin (1969).

102. M. FELDMAN, R.J. McCANN: Monge's transport problem on a Riemannian manifold. Trans. Amer. Math. Soc., 354 (2002), 1667-1697.

103. S. FINZI VITA: Numerical shape optimization for relaxed Dirichlet problems. Preprint Università di Roma "La Sapienza", Roma (1990).

104. S. FINZI VITA: Constrained shape optimization for Dirichlet problems: discretization via relaxation. Preprint Università di Roma "La Sapienza", 42 (1996).

105. G. A. FRANCFORT, F. MURAT: Homogenization and optimal bounds in linear elasticity. Arch. Rational Mech. Anal., 94 (1986), 307-334.

106. W. GANGBO, R. J. McCANN: The geometry of optimal transportation. Acta Math., 177 (1996), 113-161.

107. E. GIUSTI: Minimal Surfaces and Functions of Bounded Variation. Birkhäuser, Boston (1984).

108. E. GIUSTI: Metodi diretti nel calcolo delle variazioni. Unione Matematica Italiana, Bologna (1994).

109. F. GOLAY, P. SEPPECHER: Locking materials and the topology of optimal shapes. Eur. J. Mech. A Solids, 20 (4) (2001), 631-644.

110. H. H. GOLDSTINE: A History of the Calculus of Variations from the 17th through the 19th Century. Springer-Verlag, Heidelberg (1980).

111. P. GUASONI: Problemi di ottimizzazione di forma su classi di insiemi convessi. Tesi di Laurea, Università di Pisa, 1995-1996.

112. M. HAYOUNI: Sur la minimisation de la première valeur propre du laplacien. C. R. Acad. Sci. Paris Sér. I Math., 330 (2000), 551-556.

113. A. HENROT: Continuity with respect to the domain for the Laplacian: a survey. Control and Cybernetics, 23 (1994), 427-443.

114. A. HENROT, M. PIERRE: Optimisation de forme (book in preparation).

115. D. HORSTMANN, B. KAWOHL, P. VILLAGGIO: Newton's aerodynamic problem in the presence of friction. Preprint University of Cologne, Cologne (2000).

116. B. KAWOHL, L. TARTAR, O. PIRONNEAU, J.-P. ZOLESIO: Optimal Shape Design. Springer-Verlag, Berlin, 2001.

117. R. V. KOHN, G. STRANG: Optimal design and relaxation of variational problems, I,II,III. Comm. Pure Appl. Math., 39 (1986), 113-137, 139-182, 353-377.

118. R. V. KOHN, M. VOGELIUS: Relaxation of a variational method for impedance computed tomography. Comm. Pure Appl. Math., 40 (1987), 745777.

119. T. LACHAND-ROBERT, M.A. PELETIER: An example of non-convex minimization and an application to Newton's problem of the body of least resistance. Ann. Inst. H. Poincaré Anal. Non Linéaire, 18 (2001), 179-198

120. T. LACHAND-ROBERT, M.A. PELETIER: Newton's problem of the body of minimal resistance in the class of convex developable functions. Math. Nachr., 226 (2001), 153-176.

121. E. H. LIEB: On the lowest eigenvalue of the Laplacian for the intersection of two domains. Invent. Math., 74, (1983), 441-448.

122. P. L. LIONS: The concentration-compactness principle in the Calculus of Variations. The locally compact case, part 1. Ann. Inst. Poincaré, 1, (1984), 109-145.

123. W. LIU, P. NEITTAANMAKI, D. TIBA: Sur les problèmes d'optimisation structurelle. C. R. Acad. Sci. Paris, I-331 (2000), 101-106. 
124. K. A. LURIE, A. V. CHERKAEV: G-closure of a Set of Anisotropically Conductivity Media in the Two-Dimensional Case. J. Optimization Theory Appl., 42 (1984), 283-304.

125. K. A. LURIE, A. V. CHERKAEV: G-closure of Some Particular Sets of Admissible Material Characteristics for the Problem of Bending of Thin Elastic Plates. J. Optimization Theory Appl., 42 (1984), 305-316.

126. P. MARCELLINI: Nonconvex integrals of the calculus of variations. Methods of nonconvex analysis (Varenna, 1989), 16-57, Lecture Notes in Math. 1446, Springer-Verlag, Berlin (1990).

127. V. G. MAZ'JA: Sobolev Spaces. Springer-Verlag, Berlin (1985).

128. R. J. McCANN: Existence and uniqueness of monotone measure-preserving maps. Duke Math. J., 80 (1995), 309-323.

129. A. MIELE: Theory of Optimum Aerodynamic Shapes. Academic Press, New York (1965).

130. G. MONGE: Memoire sur la Theorie des Déblais et des Remblais. Historie de l'Académie Royale des Sciences de Paris, avec les Mémoires de Mathématique et de Physique pour la Même année, (1781), 666-704.

131. F. MORGAN: Geometric Measure Theory, a Beginners Guide. Academic Press, New York (1988).

132. F. MORGAN, R. BOLTON: Hexagonal economic regions solve the location problem. Amer. Math. Monthly, 109 (2001), 165-172.

133. C. B. MORREY: Multiple integrals in the calculus of variations. Springer, Berlin (1966).

134. U. MOSCO: Convergence of convex sets and of solutions of variational inequalities. Adv. in Math., 3 (1969), 510-585.

135. U. MOSCO: Composite media and asymptotic Dirichlet forms. J. Funct. Anal., 123 (1994), 368-421.

136. F. MURAT, J. SIMON: Sur le contrôle par un domaine géometrique. Preprint 76015, Univ. Paris VI, (1976).

137. F. MURAT, L. TARTAR: Calcul des variations et homogénéisation. Proceedings of "Les Méthodes de l'homogénéisation: Théorie et applications en physique", Ecole d'Eté d'Analyse Numérique C.E.A.-E.D.F.-INRIA, Bréausans-Nappe 1983, Collection de la direction des études et recherches d'electricité de France 57, Eyrolles, Paris, (1985), 319-369.

138. F. MURAT, L. TARTAR: Optimality conditions and homogenization. Proceedings of "Nonlinear variational problems", Isola d'Elba 1983, Res. Notes in Math. 127, Pitman, London, (1985), 1-8.

139. P. NEITTAANMAKI, D. TIBA: Shape optimization in free boundary systems. Free boundary problems: theory and applications, II (Chiba, 1999), 334-343, GAKUTO Internat. Ser. Math. Sci. Appl., 14, Gakkōtosho, Tokyo (2000).

140. F. OTTO: The geometry of dissipative evolution equations: the porous medium equation. Comm. Partial Differential Equations, 26 (2001), 101-174.

141. E. OUDET: Shape Optimization and Control. Ph.D thesis, ULP Strasbourg, France. In preparation.

142. O. PIRONNEAU: Optimal Shape Design for Elliptic Systems. Springer-Verlag, Berlin (1984).

143. G. POLYA: On the characteristic frequencies of a symmetric membrane, Math. Zeit., 63 (1955), 331-337. 
144. S. T. RACHEV, L. RÜSCHENDORF: Mass transportation problems. Vol. I Theory, Vol. II Applications. Probability and its Applications, Springer-Verlag, Berlin (1998).

145. J. SOKOLOWSKI, A. ZOCHOVSKI: On the topological derivative in shape optimization. SIAM J. Control Optim., 37 (1999), 1251-1272

146. J. SOKOLOWSKI, J.-P. ZOLESIO: Introduction to shape optimization. Shape sensitivity analysis. Springer Series in Computational Mathematics, 16. Springer-Verlag, Berlin (1992).

147. V. ŠVERÁK: On optimal shape design. J. Math. Pures Appl., 72 (1993), 537551.

148. L. TARTAR: Estimations Fines des Coefficients Homogénéises. Ennio De Giorgi Colloquium, Edited by P.Krée, Res. Notes in Math. 125, Pitman, London (1985), 168-187.

149. L. TARTAR: An introduction to the homogenization method in optimal design. In "Optimal Shape Design", Lecture Notes in Math. 1740, Springer-Verlag, Berlin (2000), 47-156.

150. R. TOADER: Wave equation in domains with many small obstacles. Asymptot. Anal., 23 (2000), 273-290.

151. J. URBAS: Mass transfer problems. Unpublished manuscript.

152. N. VAN GOETHEM: Variational problems on classes of convex domains. Preprint Dipartimento di Matematica Università di Pisa, Pisa (2000).

153. S. VENTURINI: Derivation of distance functions in $\mathbb{R}^{N}$. Preprint Dipartimento di Matematica, Università di Bologna (1991).

154. C. VILLANI: Topics in mass transportation. Book in preparation.

155. A. WAGNER: A remark on Newton's resistance formula. Preprint University of Cologne, Cologne (1998).

156. S. A. WOLF, J. B. KELLER: Range of the first two eigenvalues of the Laplacian. Proc. Roy. Soc. Lond., A-447 (1994), 397-412. 